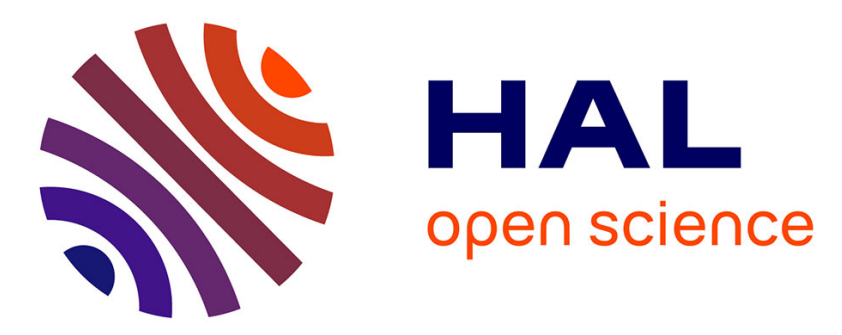

\title{
Direct and inverse energy cascades in a forced rotating turbulence experiment
}

Antoine Campagne, Basile Gallet, Frédéric Moisy, Pierre-Philippe Cortet

\section{To cite this version:}

Antoine Campagne, Basile Gallet, Frédéric Moisy, Pierre-Philippe Cortet. Direct and inverse energy cascades in a forced rotating turbulence experiment. Physics of Fluids, 2014, 26, pp.125112. 10.1063/1.4904957 . cea-01409191

\section{HAL Id: cea-01409191 https://hal-cea.archives-ouvertes.fr/cea-01409191}

Submitted on 30 Mar 2018

HAL is a multi-disciplinary open access archive for the deposit and dissemination of scientific research documents, whether they are published or not. The documents may come from teaching and research institutions in France or abroad, or from public or private research centers.
L'archive ouverte pluridisciplinaire HAL, est destinée au dépôt et à la diffusion de documents scientifiques de niveau recherche, publiés ou non, émanant des établissements d'enseignement et de recherche français ou étrangers, des laboratoires publics ou privés. 


\title{
Direct and inverse energy cascades in a forced rotating turbulence experiment
}

\author{
Antoine Campagne, ${ }^{1}$ Basile Gallet, ${ }^{1,2}$ Frédéric Moisy, ${ }^{1}$ \\ and Pierre-Philippe Cortet $^{1}$ \\ ${ }^{1}$ Laboratoire FAST, CNRS, Université Paris-Sud, 91405 Orsay, France \\ ${ }^{2}$ Laboratoire SPHYNX, Service de Physique de l'État Condensé, DSM, CEA Saclay, CNRS, \\ 91191 Gif-sur-Yvette, France
}

(Received 24 June 2014; accepted 11 December 2014; published online 31 December 2014)

\begin{abstract}
We present experimental evidence for a double cascade of kinetic energy in a statistically stationary rotating turbulence experiment. Turbulence is generated by a set of vertical flaps, which continuously injects velocity fluctuations towards the center of a rotating water tank. The energy transfers are evaluated from two-point third-order three-component velocity structure functions, which we measure using stereoscopic particle image velocimetry in the rotating frame. Without global rotation, the energy is transferred from large to small scales, as in classical three-dimensional turbulence. For nonzero rotation rates, the horizontal kinetic energy presents a double cascade: a direct cascade at small horizontal scales and an inverse cascade at large horizontal scales. By contrast, the vertical kinetic energy is always transferred from large to small horizontal scales, a behavior reminiscent of the dynamics of a passive scalar in two-dimensional turbulence. At the largest rotation rate, the flow is nearly two-dimensional, and a pure inverse energy cascade is found for the horizontal energy. To describe the scale-by-scale energy budget, we consider a generalization of the Kármán-Howarth-Monin equation to inhomogeneous turbulent flows, in which the energy input is explicitly described as the advection of turbulent energy from the flaps through the surface of the control volume where the measurements are performed. (C) 2014 AIP Publishing LLC. [http://dx.doi.org/10.1063/1.4904957]
\end{abstract}

\section{INTRODUCTION}

Global rotation is a key ingredient of many geophysical and astrophysical flows. Through the action of the Coriolis force, rotating turbulence tends to approach two-dimensionality, i.e., invariance along the rotation axis (hereafter denoted as the vertical axis by convention). ${ }^{1-3}$ Energetic 2D and 3D flow features therefore coexist in rotating turbulence, and the question of the direction of the energy cascade between spatial scales naturally arises: In 3D, energy is transferred from large to small scales ${ }^{1,2,4}$ whereas it is transferred from small to large scales in 2D, as first proposed by Kraichnan. ${ }^{5-7}$ In rotating turbulence, energy transfers depend on the Rossby number $R o$, which compares the rotation period $\Omega^{-1}$ to the turbulent turnover time. In the limit of small Ro, the fluid motions evolving on a time scale much slower than the rotation period $\Omega^{-1}$ are $2 \mathrm{D} 3 \mathrm{C}$ (two-dimensional, three-component), a result known as the Taylor-Proudman theorem, while the faster motions of frequency up to $2 \Omega$ are in the form of 3D inertial waves. ${ }^{8}$ In this limit, 3D energy transfers occur through resonant and quasi-resonant triadic interactions of inertial waves, ${ }^{9-13}$ which drive energy in a direct cascade, with a net transfer towards slow, small-scale, nearly 2D modes. ${ }^{10,14,15}$ Exactly resonant triads cannot however drive energy from 3D modes to the exactly 2D mode. In the limit of vanishing Rossby number, only those exact resonances are efficient, so the 2D3C mode is autonomous: ${ }^{16}$ It follows a purely 2D dynamics unaffected by rotation, with an inverse cascade of horizontal energy and a passive-scalar mixing of the vertical velocity. ${ }^{4,7}$ This decoupling implies that, if energy is supplied to the 3D modes only, the $2 \mathrm{D}$ mode should not be excited and no inverse cascade should be observed. 
In contrast with this asymptotic limit, most experiments and numerical simulations correspond to moderate Rossby numbers. They exhibit the emergence of large-scale columnar structures, which suggests a net transfer from the 3D "wave" modes to the $2 \mathrm{D} 3 \mathrm{C}$ "vortex" mode. ${ }^{17-25}$ For such moderate Rossby numbers, near-resonant triadic interactions, which are increasingly important as $R o$ is increased, allow for non-vanishing energy transfers between 3D and 2D modes, ${ }^{10,19,20,26,27}$ thus providing a mechanism for the emergence of inverse energy transfers: once energy is transferred to the 2D vortex mode, local 2D interactions are expected to build an upscale energy cascade. Even for a purely $3 \mathrm{D}$ forcing, the vortex mode grows as a result of near-resonant triads involving one 2D mode, and two large-vertical-scale and small-horizontal-scale 3D modes: ${ }^{20}$ this vortex mode then triggers inverse energy transfers between purely 2D modes. This intermediate Rossby number regime is of first practical interest: the Rossby number of most laboratory experiments and geophys$\mathrm{ical} /$ astrophysical flows is indeed of the order of $10^{-1}-10^{-2}$. In these situations, a natural question is to what extent direct and inverse cascades may coexist, and what sets their relative amplitudes as the Rossby number is varied.

Inverse energy cascade in rotating turbulence has been mostly investigated numerically, in the simplified configuration of a body force acting at an intermediate wave number $k_{f}$ in a periodic box..$^{11,22,25,26,28-32}$ In this setup, the inverse cascade is manifested through a growth of the energy spectrum, and hence an inverse spectral transfer, at wave numbers $k_{\perp}<k_{f}$ (with $k_{\perp}$, the wave number component normal to the rotation axis). As for $2 \mathrm{D}$ turbulence, the kinetic energy increases during this transient regime, until energetic domain size structures are formed ${ }^{33}$ or additional large-scale dissipation comes into play. Although much weaker, an inverse transfer of energy is also found in numerical simulations of decaying rotating turbulence. ${ }^{19,34,35}$ Overall, these simulations indicate that, in addition to the Rossby number, the nature of the forcing, in particular, its dimensionality ( $2 \mathrm{D}$ vs. $3 \mathrm{D})$, componentality ( $2 \mathrm{C}$ vs. $3 \mathrm{C}$ ), and helicity content, plays a key role for the existence and intensity of the inverse cascade. ${ }^{27,29}$ In addition, since shallow domains resemble 2D systems, which enhance the inverse cascade, another key parameter in this problem is the vertical confinement: the critical Rossby number under which the inverse cascade appears increases as the ratio of the box height to the forcing scale gets smaller. ${ }^{11,30,31}$

Although these numerical simulations have provided valuable insight about the conditions under which an inverse cascade takes place in rotating turbulence, the most common assumptions of homogeneity and narrow-band spectral forcing are of limited practical interest. More general forcing functions are considered in the simulations of Bourouiba et al. ${ }^{20}$ with energy input either in a large range of vertical scales and a single horizontal scale, or vice-versa. In most flows encountered in the laboratory and in geophysical/astrophysical contexts, energy injection in a given control volume is broadband and results from the spatial gradients of turbulent energy. As a consequence, the well-separated inverse and direct cascades obtained in numerical simulations with a separating wave number fixed at the forcing wave number $k_{f}$ are not relevant to describe real flows with boundary forcing. Furthermore, flows of geophysical relevance can often be considered to be in statistically steady state. Such stationary states are easily achieved in laboratory experiments, whereas they generally correspond to prohibitively long integration times for numerical simulation. This provides another justification for considering the problem of the energy cascade directions of rotating turbulence experimentally.

We therefore built an experiment aimed at studying such stationary rotating turbulence. Designing a rotating turbulence experiment which unambiguously exhibits an inverse cascade is however difficult for several reasons. First, in a statistically steady turbulence experiment, an inverse cascade can be identified only from measurements of energy transfers, i.e., from third-order velocity correlations. These measurements require very large data sets from advanced image-based diagnostic such as stereoscopic particle image velocimetry (PIV). ${ }^{36}$ Second, it is possible to separate the scale-by-scale energy fluxes from the spatial transport of energy only under the assumption of weak inhomogeneity of the flow, which is difficult to satisfy with boundary forced experiments.

Because of these difficulties, experimental evidence of inverse cascade in rotating turbulence is scarce. Indirect evidence was first provided by Baroud et al. ${ }^{37}$ in forced turbulence and later by Morize et al. $^{38}$ in decaying turbulence. In both experiments, it is reflected in a change of sign of the third-order moment of the longitudinal velocity increments in the plane normal to 
the rotation axis, $S_{3}\left(r_{\perp}\right)=\left\langle\left(\delta u_{L}\right)^{3}\right\rangle$ (where $\delta u_{L}$ is the velocity increment projected along the horizontal separation $\left.\mathbf{r}_{\perp}\right)$. Simple relations between $S_{3}\left(r_{\perp}\right)$ and the energy flux exist only either in the 3D3C (three-dimensional, three-component) isotropic case or in the 2D2C (two-dimensional, two-component) isotropic case, but not in the general axisymmetric case, so the change of sign of $S_{3}$ cannot be unambiguously related to inverse energy transfers in these experiments. More recently, evidence of inverse energy transfers has been reported by Yarom et al..${ }^{39}$ from the transient evolution of the energy spectrum in a forced rotating turbulence experiment. However, because of the unstationary and inhomogeneous nature of their experiment, it is delicate to distinguish the scale-by-scale energy transfers at a given spatial location from the spatial energy transport from the turbulence production device to the measurement area. In all these experiments, the aspect ratio is of order unity, so the 2D features of turbulence are essentially due to rotation and not confinement. The extreme case of rotating shallow water experiments is indeed known to produce a purely $2 \mathrm{D}$ dynamics with an inverse energy cascade even at small rotation rate (see, e.g., Afanasyev and Craig ${ }^{40}$ ). The integral scale measurements of van Bokhoven et al. ${ }^{41}$ in which both the fluid height and the rotation rate are varied, also confirm the combined roles of these two parameters in the generation of large-scale quasi-2D vortices.

In this paper, we investigate the interplay between direct and inverse energy cascades in a statistically stationary rotating turbulence experiment from direct measurements of scale-by-scale energy transfers. Turbulence is generated by a set of vertical flaps which continuously inject velocity fluctuations towards the center of a rotating water tank. The flaps are vertically invariant, but instabilities in their vicinity induce 3D turbulent fluctuations, so the forcing injects energy both in the 2D and $3 \mathrm{D}$ modes. We compute the energy transfers from the divergence of the two-point third-order velocity structure functions extracted from stereoscopic particle image velocimetry measurements in the rotating frame. We observe the emergence of a double cascade of energy, direct at small scales and inverse at large scales, the extension and magnitude of the inverse cascade increasing with global rotation. This overall behavior of the total kinetic energy is the superposition of different behaviors for the horizontal and vertical velocities: for rapid global rotation, the horizontal energy exhibits an inverse cascade, whereas the vertical energy follows a direct cascade. The inverse cascade of horizontal energy is found only at large scale for moderate rotation rate but gradually spreads down to the smallest scales as the rotation rate is increased. These findings are compatible with a $2 \mathrm{D} 3 \mathrm{C}$ dynamics at large rotation rate, with the horizontal velocity following a $2 \mathrm{D}$ dynamics and the vertical velocity behaving as a passive scalar.

The energy transfers in homogeneous (but not necessarily isotropic) turbulence can be described in the physical space using the Kármán-Howarth-Monin (KHM) equation. ${ }^{4,36,42}$ This approach holds for homogeneous decaying turbulence and for stationary turbulence forced by a homogeneous body force. However, it breaks down in boundary-forced experiments, in which inhomogeneities induce a transport of kinetic energy from the forcing region to the region where measurements are performed. Extended versions of the KHM equation including the effects of inhomogeneities have been proposed and proved useful to describe the energy budget in simple configurations, e.g., in wind-tunnel experiments. ${ }^{43-47}$ Here, we make use of the inhomogeneous generalization of the KHM equation proposed by Hill. ${ }^{44}$ The measurement of the different terms of this equation in the case of the largest rotation rate, which is closer to the asymptotic 2D3C state, allows us to clarify the effect of the inhomogeneous forcing in this experiment.

\section{EXPERIMENTAL SETUP}

The experimental setup is similar to the one described in Gallet $e t$ al. ${ }^{48}$ and only the features specific to the present experiments are described in detail here. The setup consists of a glass tank of $125 \times 125 \mathrm{~cm}^{2}$ square base and $65 \mathrm{~cm}$ height, filled with $50 \mathrm{~cm}$ of water and mounted on a precision rotating platform of $2 \mathrm{~m}$ diameter (see Fig. 1(a)). We have carried out experiments at five rotation rates $\Omega$ in the range from 0.21 to $1.68 \mathrm{rad} \mathrm{s}^{-1}(2-16 \mathrm{rpm})$, together with a reference experiment without rotation $(\Omega=0)$. The rotation rate is constant to better than $10^{-3}$ relative fluctuations. In the central region of the tank, we use a glass lid to avoid the paraboloidal deformation of the free surface and to allow for visualization from above. This lid is $43 \mathrm{~cm}$ above the bottom of the tank. 

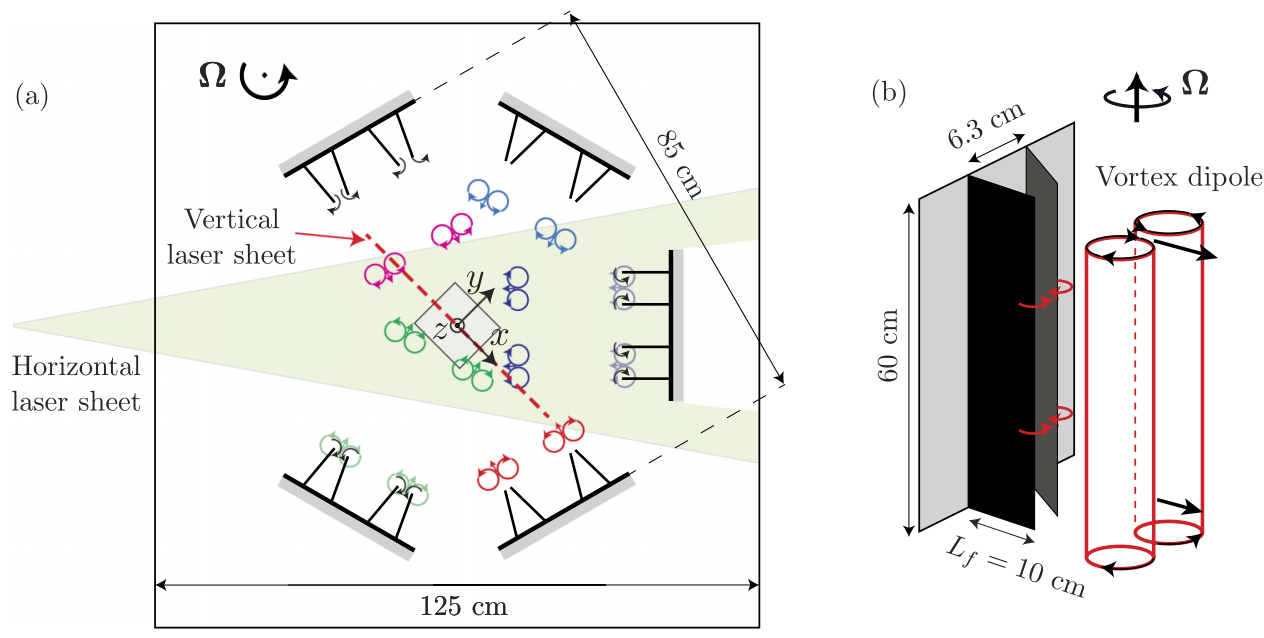

FIG. 1. (a) Schematic of the experiment: An arena of 10 pairs of vertical flaps is placed in a parallelepipedic water tank rotating at angular velocity $\Omega$. The rotation vector $\Omega$ is vertical and the system is viewed from above. The rectangle at the center of the arena indicates the horizontal region where 2D-3C velocity fields are measured by stereoscopic particle image velocimetry. The drawing shows idealized vortex dipoles emitted by the generators, before they interact in the center of the arena. (b) Perspective view of a pair of flaps.

A statistically stationary turbulent flow is produced by a set of ten vortex dipole generators. They are arranged in 5 blocks of 2 generators located around a hexagonal arena of $85 \pm 5 \mathrm{~cm}$ width, each of them being oriented towards the center of the arena (Fig. 1(a)). One side of the hexagon is left open to illuminate the center of the arena with a horizontal laser sheet. This forcing device was initially designed to generate turbulence in stratified fluids and is described in detail in Refs. 49 and 50. Each generator consists of a pair of vertical flaps, $60 \mathrm{~cm}$ high and $L_{f}=10 \mathrm{~cm}$ long, each flap rotating about one of its vertical edges (Fig. 1(b)). Thanks to DC motors and a system of gears and cams, the pairs of flaps are driven in a periodic motion of $8.5 \mathrm{~s}$ duration and $9^{\circ}$ amplitude: the two flaps being initially parallel, they first rotate with an angular velocity $\omega_{f}=0.092 \mathrm{rad} \mathrm{s}^{-1}$ during $1.7 \mathrm{~s}$ until their tips almost touch each other. They remain motionless during $3.4 \mathrm{~s}$, before reopening during $1.7 \mathrm{~s}$ until they reach the initial parallel configuration again. They finally remain motionless during the last $1.7 \mathrm{~s}$ of the cycle. The motions of the two adjacent pairs of flaps of a given block are in phase, but an arbitrary phase shift is set between the five blocks. The rotation of the platform is set long before the start of this forcing device, at least $1 \mathrm{~h}$, in order for transient spin-up recirculations to be damped. Once solid-body rotation is reached, we start the generators, and a statistically stationary state is reached in the center of the tank after a few minutes.

The Reynolds number based on the flap length $L_{f}$ and flap angular velocity $\omega_{f}$ is $R e_{f}=\omega_{f} L_{f}^{2} / v$ $=920$. The flow generated by the closing of the flaps consists of an initially vertically invariant vortex dipole (Fig. 1(a)) which quickly becomes unstable and produces small-scale 3D turbulent fluctuations. This turbulent burst self-advects towards the center of the arena because of the persistent large-scale vortex dipole component. The Rossby number based on the flap angular velocity is low, $R o_{f}=\omega_{f} / 2 \Omega \in[0.03,0.22]$ (see Table I), indicating that the flow generated by the flap motion is influenced by rotation right from the generators (except for the non-rotating experiment). Turbulence in the center of the flow can be also characterized locally by the turbulent Reynolds and Rossby numbers based on the r.m.s. velocity $u_{\mathrm{rms}}$ and the horizontal integral scale $L_{\perp}$ defined in Eq. (4) (see Sec. IV A): $R e=u_{\mathrm{rms}} L_{\perp} / v$ and $R o=u_{\mathrm{rms}} / 2 \Omega L_{\perp}$ (values are given in Table I).

The three components of the velocity field $\mathbf{u}(\mathbf{x}, t)=\left(u_{x}, u_{y}, u_{z}\right)$ are measured in a horizontal and a vertical plane in the rotating frame (Fig. 1(a)) using a stereoscopic particle image velocimetry (PIV) system. ${ }^{51,52}$ The two regions of interest are centered with respect to the arena of generators. It is a square of about $14 \times 14 \mathrm{~cm}^{2}$ in a vertical plane along the diagonal of the base of the tank and a square of $12 \times 12 \mathrm{~cm}^{2}$ in a horizontal plane at mid-depth. The flow is seeded with $10 \mu \mathrm{m}$ tracer particles and illuminated by a laser sheet generated by a double $140 \mathrm{~mJ} \mathrm{Nd}$ :YAG pulsed 
TABLE I. Flow parameters for the different rotation rates $\Omega$ : Rossby number based on the flap velocity $R o_{f}$, rate of turbulence $k / K$, inhomogeneity factor $\gamma$, horizontal integral scale $L_{\perp}$, turbulent Reynolds number $R e$, and Rossby number $R o$. These figures are computed from the stereoscopic PIV data in the horizontal plane (Fig. 1(a)). See text for definitions.

\begin{tabular}{lcccccc}
\hline \hline$\Omega(\mathrm{rpm})$ & 0 & 2 & 4 & 8 & 12 & 16 \\
\hline$R o_{f}$ & $\infty$ & 0.22 & 0.11 & 0.055 & 0.037 & 0.028 \\
$k / K$ & 0.48 & 0.79 & 0.89 & 0.95 & 0.94 & 0.97 \\
$\gamma$ & 0.17 & 0.15 & 0.06 & 0.05 & 0.04 & 0.10 \\
$L_{\perp}(\mathrm{mm})$ & 24 & 43 & 45 & 44 & 42 & 38 \\
$R e$ & 140 & 230 & 350 & 420 & 400 & 330 \\
$R o$ & $\infty$ & 0.30 & 0.20 & 0.13 & 0.087 & 0.068 \\
\hline \hline
\end{tabular}

laser mounted on the rotating platform (Fig. 1(a)). The illuminated flow section is imaged with two double-buffer cameras aiming at the laser sheet under different incidence angles. Images are taken from above through the glass lid for the measurements in the horizontal plane and from two adjacent vertical sides of the tank for the measurements in the vertical plane.

Each acquisition consists of 3600 quadruplets of images (one pair per camera) recorded at $0.35 \mathrm{~Hz}$ with a $50 \mathrm{~ms}$ time lag between the two images of a given pair. The 3 velocity components are computed in the two-dimensional measurement plane using stereoscopic reconstruction. The cross-correlations are based on interrogation windows of $32 \times 32$ pixels with $50 \%$ overlap. The resulting $2 \mathrm{D} 3 \mathrm{C}$ velocity fields are sampled on a grid of $105 \times 105$ (respectively, $80 \times 80$ ) vectors with a spatial resolution of $1.15 \mathrm{~mm}$ (respectively, $1.75 \mathrm{~mm}$ ) in the horizontal (respectively, vertical) plane.

\section{LOCAL HOMOGENEITY AND AXISYMMETRY}

In this experiment, kinetic energy is injected by the generators located around the region of interest, so an inward transport of energy takes place from the generators to the center of the arena. An important feature of turbulence in this configuration is the presence or not of a mean flow induced by the generators: this indicates whether the transport of energy can be mainly attributed to a reproducible flow or to turbulent fluctuations. This can be addressed by performing a Reynolds decomposition of the velocity field

$$
\mathbf{u}(\mathbf{x}, t)=\overline{\mathbf{u}}(\mathbf{x})+\mathbf{u}^{\prime}(\mathbf{x}, t),
$$

where $\overline{\mathbf{u}}(\mathbf{x})$ is the time-averaged velocity field and $\mathbf{u}^{\prime}(\mathbf{x}, t)$ its turbulent part. From this decomposition, we can compute the turbulent and total kinetic energies, $k=\left\langle\overline{\left\langle\mathbf{u}^{\prime}(\mathbf{x}, t)^{2}\right.}\right\rangle_{\mathbf{x}} / 2$ and $K=\left\langle\overline{\mathbf{u}(\mathbf{x}, t)^{2}}\right\rangle_{\mathbf{x}} / 2$, respectively, where $\langle\cdot\rangle_{\mathbf{x}}$ is a spatial average over the horizontal region of interest. The turbulence rate $k / K$ is about $50 \%$ in the non-rotating experiment but rapidly increases up to $97 \%$ as the rotation rate $\Omega$ is increased (see Table I) indicating that, under rotation, the turbulent structures are essentially self-advected from the generators towards the center of the arena. In the following, we therefore focus on the turbulent component $\mathbf{u}^{\prime}(\mathbf{x}, t)$ which dominates the flow in the rotating case.

Although turbulence is necessarily inhomogeneous in this configuration, with more energy near the generators than at the center of the flow, we may expect a reasonable local homogeneity in the measurement area because of its small size (square of about $13 \mathrm{~cm}$ side) compared to the distance to the generators ( $33 \mathrm{~cm}$ from the center of the arena to the tip of the flaps). Before investigating the scale-by-scale energy distribution and energy transfers from spatially averaged two-point statistics, it is therefore important to quantify the degree of homogeneity of the flow. Since most of the energy is turbulent, we can quantify the level of homogeneity by the spatial standard deviation of the turbulent kinetic energy

$$
\gamma=\frac{\left\langle[k(\mathbf{x})-k]^{2}\right\rangle_{\mathbf{x}}^{1 / 2}}{k}
$$


with $k(\mathbf{x})=\overline{\mathbf{u}^{\prime}(\mathbf{x}, t)^{2}} / 2$ the local time-averaged turbulent energy (such that $k=\langle k(\mathbf{x})\rangle_{\mathbf{x}}$ ). This ratio is given in Table I. It is smaller than $10 \%$ for $\Omega>4 \mathrm{rpm}$, indicating a reasonable degree of homogeneity in the region of interest.

A last single-point quantity of interest to characterize the turbulence field in this configuration is the spatially averaged velocity correlation tensor, $\left\langle\overline{u_{i}^{\prime} u_{j}^{\prime}}\right\rangle_{\mathbf{x}}$ (the trace of this tensor is twice the turbulence kinetic energy). For axisymmetric turbulence with respect to $z$, one has $\left\langle\overline{u_{x}^{\prime 2}}\right\rangle_{\mathbf{x}}=\left\langle\overline{u_{y}^{\prime 2}}\right\rangle_{\mathbf{x}} \neq$ $\left\langle\overline{u_{z}^{\prime 2}}\right\rangle_{\mathbf{x}}$ (i.e., turbulence is isotropic in the horizontal plane), with zero non-diagonal components. For 3D isotropic turbulence, the three diagonal components are equal (i.e., $\left\langle\overline{u_{i}^{\prime} u_{j}^{\prime}}\right\rangle_{\mathbf{x}}=\frac{2}{3} k \delta_{i j}$ ). This tensor therefore characterizes the componentality of turbulence, i.e., the isotropy with respect to the velocity components. It must not be confused with the dimensionality of turbulence, which characterizes the dependence of the two-point velocity statistics with respect to orientation of the separation vector joining the two points (investigated in Sec. IV).

In Fig. 2, we see that turbulence is nearly axisymmetric, with $\left\langle\overline{u_{x}^{\prime 2}}\right\rangle_{\mathbf{x}} \simeq\left\langle\overline{u_{y}^{\prime 2}}\right\rangle_{\mathbf{x}}$ to within $3 \%$ in the rotating case and $10 \%$ in the non-rotating case, and with the three non-diagonal components less than $10 \%$ of the diagonal components. As expected, turbulence is never isotropic, even in the case $\Omega=0$, for which $\left\langle\overline{u_{x}^{\prime 2}}\right\rangle_{\mathbf{x}} \simeq\left\langle\overline{u_{y}^{\prime 2}}\right\rangle_{\mathbf{x}} \simeq 2\left\langle\overline{u_{z}^{\prime 2}}\right\rangle_{\mathbf{x}}$. This anisotropy originates from the vertically invariant forcing by the flaps, which induces significantly weaker vertical velocity fluctuations than horizontal ones. As the rotation rate $\Omega$ increases, $\left\langle\overline{u_{z}^{\prime 2}}\right\rangle_{\mathbf{x}}$ remains roughly constant, whereas $\left\langle\overline{u_{x}^{\prime 2}}\right\rangle_{\mathbf{x}}$ and $\left\langle\overline{u_{y}^{\prime 2}}\right\rangle_{\mathbf{x}}$ first increase with $\Omega$ before saturating beyond $8 \mathrm{rpm}(R o \simeq 0.13)$. At large $\Omega$, the vertical kinetic energy represents about $10 \%$ of the total energy.

\section{SCALE-BY-SCALE ENERGY DISTRIBUTION AND TRANSFERS}

We now focus on the scale-by-scale energy distribution and energy transfers. For this we must use two-point quantities: let us consider two points $\mathrm{A}$ and $\mathrm{B}$ in the turbulent flow at positions $\mathbf{x}_{A}$ and $\mathbf{x}_{B}$. We define the mid-point position $\mathbf{X}=\left(\mathbf{x}_{A}+\mathbf{x}_{B}\right) / 2$ and the separation vector $\mathbf{r}=\mathbf{x}_{B}-\mathbf{x}_{A}$. Using cylindrical coordinates, the separation $\mathbf{r}$ writes $\left(r_{\perp}, \varphi, r_{\|}\right)$, with $r_{\perp}=\left(r_{x}^{2}+r_{y}^{2}\right)^{1 / 2}$ and $r_{\|}=r_{z}$. In homogeneous turbulence, all statistical averages are functions of the separation vector $\mathbf{r}$ only. However, inhomogeneity plays a key role in boundary forced experiments, and we thus consider the inhomogeneous framework in which ensemble averages remain functions of both $\mathbf{r}$ and $\mathbf{X}$.

The centered velocity increment for separation $\mathbf{r}$, mid-point $\mathbf{X}$, and time $t$ is

$$
\begin{aligned}
\delta \mathbf{u}^{\prime}(\mathbf{X}, \mathbf{r}, t) & =\mathbf{u}_{B}^{\prime}(\mathbf{X}, \mathbf{r}, t)-\mathbf{u}_{A}^{\prime}(\mathbf{X}, \mathbf{r}, t) \\
& =\mathbf{u}^{\prime}\left(\mathbf{x}_{B}=\mathbf{X}+\mathbf{r} / 2, t\right)-\mathbf{u}^{\prime}\left(\mathbf{x}_{A}=\mathbf{X}-\mathbf{r} / 2, t\right) .
\end{aligned}
$$

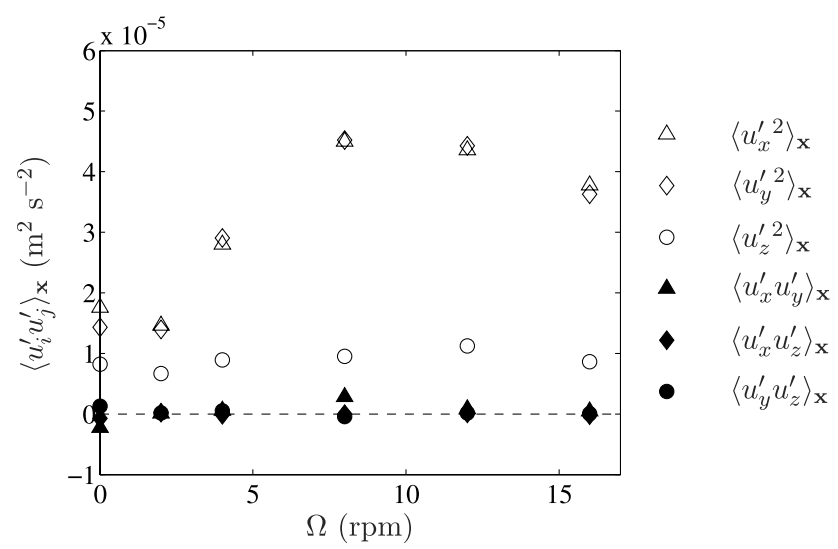

FIG. 2. Components of the velocity correlation tensor $\left\langle\overline{u_{i}^{\prime} u_{j}^{\prime}}\right\rangle_{\mathbf{x}}$ (with $\left.(i, j) \in(x, y, z)\right)$ as a function of $\Omega$ averaged over the horizontal region of interest. The non-diagonal components are nearly zero and $\left\langle\overline{u_{x}^{\prime 2}}\right\rangle_{\mathbf{x}} \simeq\left\langle\overline{u_{y}^{\prime 2}}\right\rangle_{\mathbf{x}}$, indicating statistically axisymmetric turbulence. $\Omega=0,2,4,8,12,16 \mathrm{rpm}$ corresponds to turbulent Rossby number $R o=\infty, 0.30,0.20,0.13$, $0.087,0.068$, respectively. 
To perform an energy budget in this inhomogeneous context, we first need to define a control region for the mid-point $\mathbf{X}$ : we consider only points A and B for which $\mathbf{X}$ lies in square $S_{X}$ of side $40 \mathrm{~mm}$ centered in the PIV field. The relatively small size of the square allows for the separation $|\mathbf{r}|$ to be as large as $80 \mathrm{~mm}$ with the two points A and B still lying in the PIV field. The statistical averages are defined as an average over time and over all the positions of $\mathbf{X}$ inside $S_{X}$. In the following, the spatial average $\langle.\rangle_{\mathbf{X}}$ over $\mathbf{X} \in S_{X}$ will simply be denoted by $\langle$.$\rangle .$

Defining the statistics from centered or non-centered increments would be equivalent for homogeneous turbulence, and most of the remainder of this section can be understood in this framework. However, the use of centered increments plays a key role in Sec. V where we discuss the scale-by-scale energy budget: there is a balance at every scale $\mathbf{r}$ between viscous dissipation, nonlinear transfers between different scales (flux in $\mathbf{r}$ space), and advection of kinetic energy at scale $\mathbf{r}$ through the boundaries of the domain $S_{X}$ (flux in $\mathbf{X}$ space). The latter term is the source term of the energy budget, which vanishes if turbulence is assumed to be homogeneous.

\section{A. Energy distribution}

We characterize the distribution of the turbulent energy among spatial scales by the anisotropic second-order structure function, defined as the variance of the centered velocity increments ${ }^{55}$

$$
E(\mathbf{r})=\left\langle\overline{\left(\delta \mathbf{u}^{\prime}\right)^{2}}\right\rangle .
$$

The angular average of this quantity, $\mathcal{E}(r)=1 /(4 \pi) \int_{\theta=0}^{\pi} \int_{\varphi=0}^{2 \pi} E(r, \theta, \varphi) \sin \theta d \theta d \varphi$, where $(r, \theta, \varphi)$ is the usual spherical coordinate system, can be interpreted as the energy contained in eddies of size $r$ or less, provided that $r$ is larger than the dissipative scale. ${ }^{2,53}$ For isotropic turbulence, $E(\mathbf{r})=\mathcal{E}(r)$ therefore directly measures the cumulative energy from 0 to $r$. For anisotropic turbulence, $E(\mathbf{r})$ contains in addition key information on the anisotropic distribution of energy among eddies of characteristic horizontal and vertical scale given by $r_{\perp}$ and $r_{\|}$, respectively. For isotropic turbulence, the isosurfaces of $E(\mathbf{r})$ are spherical, while for axisymmetric turbulence about the vertical they are invariants with respect to rotations around the $r_{z}$ axis. Two-dimensional turbulence would give exactly cylindrical iso- $E(\mathbf{r})$ (invariant by translation along $r_{z}$ ), which is a special case of axisymmetric turbulence.

Figure 3 shows the maps of the normalized energy distributions, $E(\mathbf{r}) / E_{0}$, in the horizontal $\left(r_{x}, r_{y}\right)$ and vertical $\left(r_{x}, r_{z}\right)$ planes for $\Omega=0$ and $16 \mathrm{rpm}$, with $E_{0}=\left\langle\overline{\mathbf{u}_{A}^{\prime 2}+\mathbf{u}_{B}^{\prime 2}}\right\rangle_{\mathbf{X}}$ taken at $\mathbf{r}=r_{\max } \mathbf{e}_{\perp}$ and $r_{\max }=80 \mathrm{~mm}$ as the maximum separation. ${ }^{56}$ In Figs. 3(a) and 3(b), the iso-contours of $E(\mathbf{r})$ are nearly circular in the horizontal plane, both without and with rotation, indicating the good level of two-point axisymmetry of turbulence. For the largest horizontal scales considered here $(|\mathbf{r}|=80 \mathrm{~mm}), E / E_{0}$ reaches 0.98 for $\Omega=0$, indicating that nearly all the turbulent energy is contained in the range of scales of interest, whereas it reaches 0.89 only at $\Omega=16 \mathrm{rpm}$, indicating that structures larger than the maximum available scale still contain energy. This is a first indication of the emergence of large horizontal structures in the presence of rotation. This effect can be further quantified by the horizontal integral scale,

$$
L_{\perp}=\int_{0}^{r^{*}} C\left(r_{\perp}\right) d r_{\perp}
$$

with $C\left(r_{\perp}\right)=1 /(2 \pi) \int_{0}^{2 \pi} C\left(\mathbf{r}_{\perp}\right) d \varphi$ and $C\left(\mathbf{r}_{\perp}\right)=2\left\langle\mathbf{u}_{A}^{\prime} \cdot \mathbf{u}_{B}^{\prime}\right\rangle_{\mathbf{X}} / E_{0}$ as the two-point correlation function. The conventional definition is such that $r^{*}=\infty$, but using here a finite truncation at $r^{*}$, chosen such that $C\left(r^{*}\right)=0.25$, is necessary because $C\left(r_{\perp}\right)$ does not reach 0 at the maximum available scale $r_{\perp}=80 \mathrm{~mm}$. Values of $L_{\perp}$ are given in Table I. In the absence of rotation, $L_{\perp} \simeq 24 \mathrm{~mm}$, which corresponds to the characteristic size of the turbulent fluctuations generated by the flaps. As $\Omega$ is increased, $L_{\perp}$ grows by nearly a factor of 2 compared to the non-rotating case, confirming the generation of large-scale structures.

We now turn to the energy distribution in the vertical plane (Figs. 3(c) and 3(d)). $E(\mathbf{r})$ is anisotropic both with and without rotation, with a trend towards vertical elongation of the contour lines at large scales. This anisotropy is, however, weak at $\Omega=0$ and affects preferentially the large 

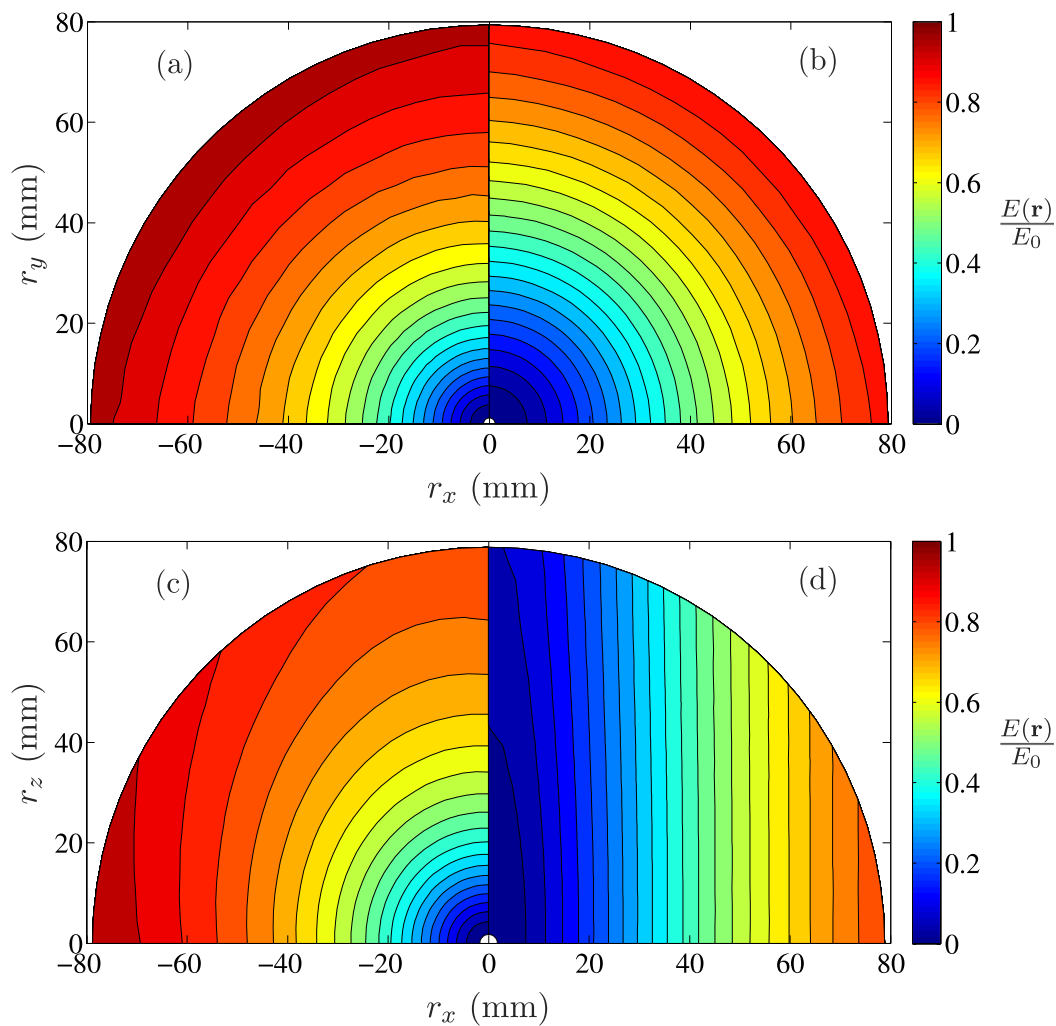

FIG. 3. Maps of the normalized energy distribution $E(\mathbf{r}) / E_{0}$, (a) and (b) in the horizontal $\left(r_{x}, r_{y}\right)$ plane, and (c) and (d) in the vertical $\left(r_{x}, r_{z}\right)$ plane. (a) and (c) correspond to the experiment with $\Omega=0(R o=\infty)$, and (b) and (d) to $\Omega=16 \mathrm{rpm}$ $(R o=0.068)$.

scales: this is a direct consequence of the vertical invariance of the forcing device, which creates a nearly $2 \mathrm{D}$ flow at large scale carrying small-scale 3D fluctuations. The anisotropy is much more pronounced in the presence of rotation and persists down to the smallest scales, indicating a trend towards quasi-2D turbulence. This scale dependence of the anisotropy can be quantified by the ratio

$$
A_{E}(r)=\frac{E_{\perp}(r)-E_{\|}(r)}{E_{\perp}(r)+E_{\|}(r)}
$$

with $E_{\perp}(r)=E\left(r_{x}=r, r_{z}=0\right)$ and $E_{\|}(r)=E\left(r_{x}=0, r_{z}=r\right)$. This ratio is zero for 3D isotropic turbulence and 1 for 2D turbulence. The plot of $A_{E}(r)$ in Fig. 4 shows a growth of anisotropy with $r$ at all rotation rates. This growth is weak for $\Omega=0$ (with $A_{E}$ increasing from -0.01 to 0.1 ), indicating that the 2D nature of the forcing has a weak influence at these scales in the center of the tank. The anisotropy is much stronger when $\Omega \neq 0: A_{E}(r)$ first grows rapidly from $r=0$ to $r \sim 10 \mathrm{~mm}$ before saturating. For the largest available rotation rate, $\Omega=16 \mathrm{rpm}$, turbulence is nearly $2 \mathrm{D}$ for $r>10 \mathrm{~mm}$, with $A_{E}(r) \simeq 0.85$, but remains significantly $3 \mathrm{D}$ at smaller scales.

\section{B. Energy transfers}

We now consider the scale-by-scale energy transfers defined from third-order moments of velocity increments. We start from the Kármán-Howarth-Monin equation for time-dependent homogeneous (but not necessarily isotropic) turbulence ${ }^{4,42}$

$$
\frac{1}{4} \partial_{t} E(\mathbf{r}, t)=-\Pi(\mathbf{r}, t)+\frac{1}{2} \nu \nabla_{\mathbf{r}}^{2} E-\epsilon,
$$




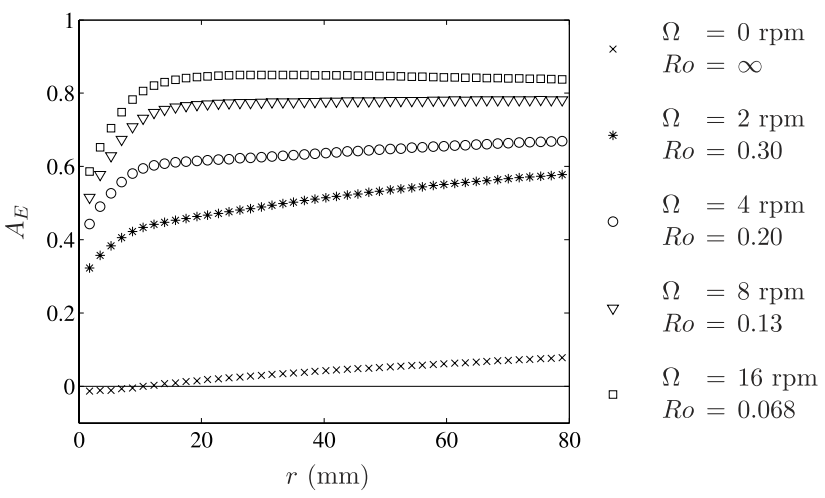

FIG. 4. Scale-dependent anisotropy factor $A_{E}(r)$ (Eq. (5)) of the energy distribution as a function of $r$ for different rotation rates $\left(A_{E}=0\right.$ for 3D isotropic turbulence and $A_{E}=1$ for 2D3C turbulence).

where $E(\mathbf{r}, t)=\left\langle\left(\delta \mathbf{u}^{\prime}\right)^{2}\right\rangle_{\mathbf{X}, E}$ and

$$
\Pi(\mathbf{r}, t)=\frac{1}{4} \nabla_{\mathbf{r}} \cdot\left\langle\left(\delta \mathbf{u}^{\prime}\right)^{2} \delta \mathbf{u}^{\prime}\right\rangle_{\mathbf{X}, E}
$$

is the energy transfer term in scale-space (with $\boldsymbol{\nabla}_{\mathbf{r}}$. the divergence with respect to the vector separation $\mathbf{r})$, and $\epsilon=v\left(\left(\partial_{i} u_{j}^{\prime}+\partial_{j} u_{i}^{\prime}\right)^{2}\right\rangle_{\mathbf{X}, E} / 2$ the instantaneous energy dissipation rate. Here, the brackets $\langle\cdot\rangle_{\mathbf{X}, E}$ represent spatial and ensemble average. Similar to the angular average $\mathcal{E}(r)$ of $E(\mathbf{r})$, which represents the cumulative energy from scale 0 to $r$, the angular average $\mathcal{P}(r)=(4 \pi)^{-1}$ $\int_{\theta=0}^{\pi} \int_{\varphi=0}^{2 \pi} \Pi(r, \theta, \varphi) \sin \theta d \theta d \varphi$ can be interpreted as the energy flux from scales smaller than $r=|\mathbf{r}|$ towards scales larger than $r$. For isotropic turbulence, the sign of $\Pi(\mathbf{r})=\mathcal{P}(r)$, therefore, gives the direction of the energy cascade, forward if $\Pi(\mathbf{r})<0$ and inverse if $\Pi(\mathbf{r})>0$. In the inhomogeneous case, additional terms corresponding to advection of energy between different regions of the turbulent flow appear in Eq. (6). In the absence of body forces, which are not relevant in our experiment, advection of energy from outside the control domain is the only source term to sustain stationary turbulence: we will come back to this point in Sec. V.

In the following, we focus on stationary turbulence, and we take $\partial_{t} E=0$ in Eq. (6). The ensemble average $\langle\cdot\rangle_{E}$ can be therefore replaced by a temporal average, which we denote as $\overline{\text {. }}$ For axisymmetric turbulence, it is convenient to decompose the flux (7) into its perpendicular (horizontal) and parallel (vertical) contributions,

$$
\begin{aligned}
\Pi(\mathbf{r}) & =\Pi_{\perp}(\mathbf{r})+\Pi_{\|}(\mathbf{r}) \\
& =\frac{1}{4} \nabla_{\perp} \cdot\left\langle\overline{\left(\delta \mathbf{u}^{\prime}\right)^{2} \delta \mathbf{u}_{\perp}^{\prime}}\right\rangle+\frac{1}{4} \nabla_{\|}\left\langle\overline{\left(\delta \mathbf{u}^{\prime}\right)^{2} \delta u_{\|}^{\prime}}\right\rangle,
\end{aligned}
$$

with $\nabla_{\perp}=\mathbf{e}_{x} \partial_{r_{x}}+\mathbf{e}_{y} \partial_{r_{y}}$ and $\nabla_{\|}=\partial_{r_{z}}$. We focus in the following on pure horizontal separations by setting $r_{\|} \equiv r_{z}=0$, and we perform an azimuthal average over $\varphi$ to improve the statistics. Both contributions from Eq. (8) are then functions of the horizontal separation $r_{\perp}$ only.

For strictly $2 \mathrm{D}$ turbulence, vertical invariance implies $\Pi_{\|}=0$. The vertical flux $\Pi_{\|}$cannot be measured here, because we cannot access the vertical derivative $\nabla_{\|}$from measurements in the horizontal plane. In principle, one could use the data in the vertical plane, but we found significant departure from axisymmetry for third-order quantities (although second-order quantities are found nearly axisymmetric, as shown in Fig. 3(a)). This lack of axisymmetry can be circumvented by performing an azimuthal average with respect to $\varphi$, which is possible in the horizontal plane only.

For the non-rotating experiment, Fig. 5 shows that the horizontal flux $\Pi_{\perp}\left(r_{\perp}\right)$ is negative at all scales, as expected for a direct energy cascade from large to small scales, with $\Pi_{\perp} \rightarrow 0$ as $r_{\perp} \rightarrow 0$ in the viscous range. The observed $10 \%$ decrease in $\left|\Pi_{\perp}\left(r_{\perp}\right)\right|$ for $r_{\perp}$ beyond $15 \mathrm{~mm}$ might be due to the vertical invariance of the forcing device: the large scales are slightly $2 \mathrm{D}$ (see Fig. 4), which may enhance the inverse energy transfers and reduce the direct ones. However, as discussed in Sec. V, boundary-driven flows display some inhomogeneity at large scale, which challenges the 


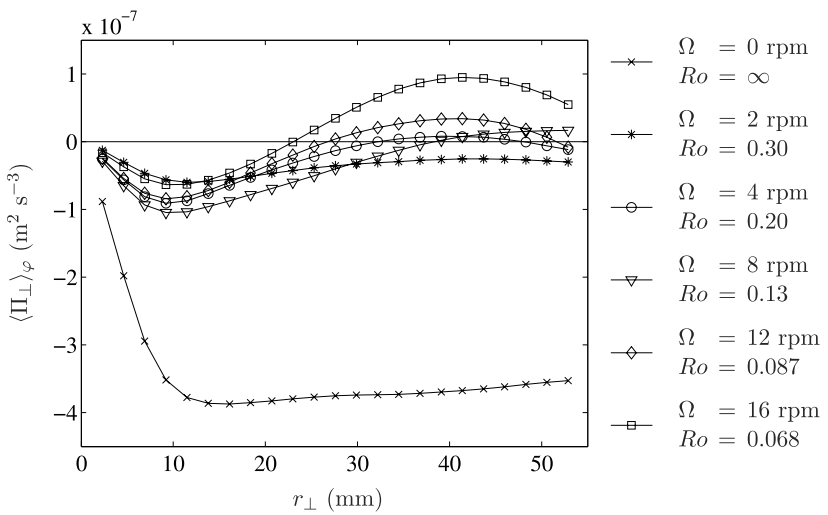

FIG. 5. Azimuthal average of the horizontal energy flux, $\left\langle\Pi_{\perp}\right\rangle_{\varphi}$, as a function of horizontal separation $r_{\perp}$ and for various rotation rates $\Omega$. These data are computed from measurements in the horizontal plane. A negative value (respectively, positive) corresponds to a direct (respectively, inverse) energy transfer.

interpretation of $\Pi_{\perp}$ in terms of pure scale-by-scale energy transfers. Therefore, we do not elaborate more on the behavior of $\Pi_{\perp}$ at scales larger than $r_{\perp}>40 \mathrm{~mm}$ (see details in Sec. V).

For increasing rotation rates, $\Pi_{\perp}\left(r_{\perp}\right)$ strongly decreases at intermediate scales in absolute value, and eventually a change of sign is observed at large scales beyond $4 \mathrm{rpm}(R o \simeq 0.2)$. This indicates the onset of an inverse energy cascade, which spreads towards smaller scales as $\Omega$ increases. Remarkably, the double cascade persists even at the largest rotation rate $(\Omega=16 \mathrm{rpm}, R o \simeq 0.068)$, with the coexistence of an inverse flux $\left(\Pi_{\perp}>0\right)$ at large scales and a direct flux $\left(\Pi_{\perp}<0\right)$ at small scales. This implies that, on average, energy must be supplied at intermediate scales (of the order of $25 \mathrm{~mm}$ for $\Omega=16 \mathrm{rpm}$ ): we return to this point in Sec. V.

\section{Horizontal transfers of horizontal and vertical energy}

The energy flux $\Pi_{\perp}\left(r_{\perp}\right)$ contains both the horizontal flux of horizontal energy, $\left(\delta \mathbf{u}_{\perp}^{\prime}\right)^{2}$, and the horizontal flux of vertical energy, $\left(\delta u_{\|}^{\prime}\right)^{2}$. To get further insight into the double cascade observed in Fig. 5, we decompose $\Pi_{\perp}$ as follows:

$$
\begin{aligned}
\Pi_{\perp}\left(r_{\perp}\right) & =\Pi_{\perp}^{(\perp)}\left(r_{\perp}\right)+\Pi_{\perp}^{(\|)}\left(r_{\perp}\right) \\
& \left.=\frac{1}{4} \boldsymbol{\nabla}_{\perp} \cdot\left\langle\overline{\left(\delta \mathbf{u}_{\perp}^{\prime}\right)^{2} \delta \mathbf{u}_{\perp}^{\prime}}\right\rangle+\frac{1}{4} \nabla_{\perp} \cdot \overline{\left\langle\left(\delta u_{\|}^{\prime}\right)^{2} \delta \mathbf{u}_{\perp}^{\prime}\right.}\right\rangle
\end{aligned}
$$

These two contributions are shown in Fig. 6. Interestingly, we observe that $\Pi_{\perp}^{(I)}$ remains negative at all rotation rates, indicating that vertical energy is always transferred from large to small horizontal scales, whereas $\Pi_{\perp}^{(\perp)}$ becomes positive as the rotation rate is increased, a signature of the onset of an inverse cascade for the horizontal energy. In the non-rotating case, this negative flux $\Pi_{\perp}^{(I I)}$ is compatible with the classical direct cascade framework of 3D turbulence. By contrast, in the presence of rotation, for the scales at which the inverse cascade of $\left(\delta \mathbf{u}_{\perp}^{\prime}\right)^{2}$ is observed, the direct cascade of $\left(\delta u_{\|}^{\prime}\right)^{2}$ is reminiscent of the behavior of a passive scalar advected by a two-dimensional flow; the stretching and folding of the vertical velocity by the horizontal flow produce small scales through filamentation, inducing a direct horizontal cascade of vertical velocity. ${ }^{7,42,54}$ We provide in Sec. V further assessment of this picture.

Figure 6 also indicates that the horizontal flux of vertical energy $\Pi_{\perp}^{(\|)}$is a significant contribution to $\Pi_{\perp}$ for all rotation rates. For the low rotation rate $\Omega=2 \mathrm{rpm}(R o=0.3)$, although a significant inverse cascade already takes place at large scale for the horizontal energy $\left(\Pi_{\perp}^{(\perp)}>0\right)$, it is hidden by a stronger direct cascade of vertical energy $\left(\Pi_{\perp}^{(\|)}<0\right)$. This results in an overall direct cascade of total energy $\left(\Pi_{\perp}<0\right)$. For larger rotation rates, the inverse cascade of horizontal energy becomes dominant, eventually leading to $\Pi_{\perp}^{(\perp)}>0$ at all scales for $\Omega \geq 12 \mathrm{rpm}$. The crossover scale 

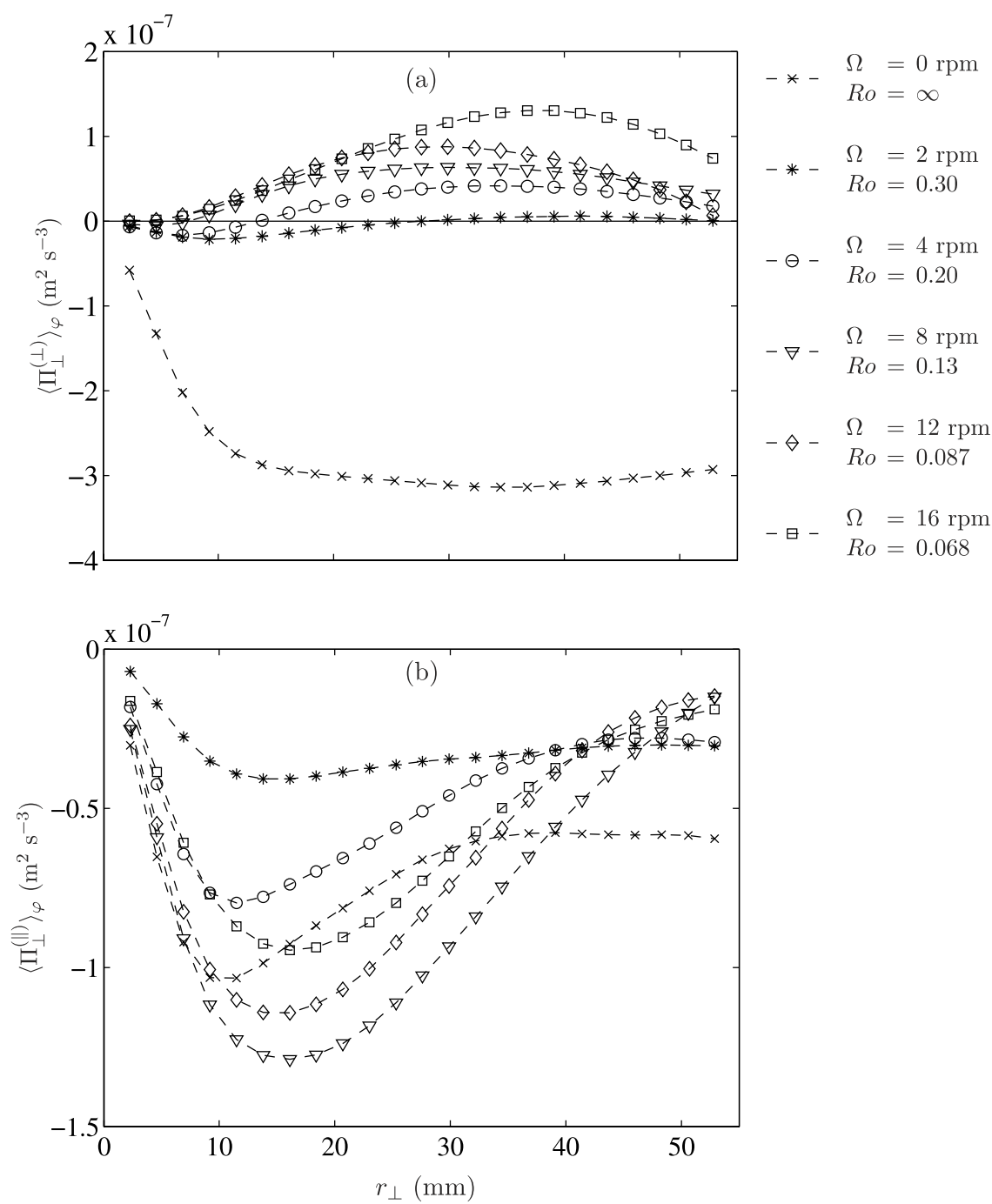

FIG. 6. Horizontal flux of (a) horizontal energy $\Pi_{\perp}^{(\perp)}\left(r_{\perp}\right)$, and (b) vertical energy, $\Pi_{\perp}^{(\|)}\left(r_{\perp}\right)$, at various rotation rates $\Omega$. A negative flux corresponds to a direct energy transfer (from large to small scales) whereas a positive flux corresponds to an inverse energy transfer.

separating the direct and inverse cascades of horizontal energy rapidly decreases as $\Omega$ increases, going from $\sim 30 \mathrm{~mm}$ for $\Omega=2 \mathrm{rpm}$ to zero for $\Omega \geq 12 \mathrm{rpm}$ (and then $\Pi_{\perp}^{(\perp)}>0$ over the whole range of scales).

\section{SCALE-BY-SCALE ENERGY BUDGET}

\section{A. Inhomogeneous Kármán-Howarth-Monin equation}

To provide a physical interpretation for the sign of the scale-by-scale energy flux $\Pi(\mathbf{r})$, we must describe carefully the energy input in the experiment and in particular, its scale dependence. In numerical simulations of homogeneous stationary turbulence, this source term usually originates from a random body force acting on a narrow range of scales. By contrast, here the fluid motion is driven by moving solid boundaries, so the energy injection in a given control volume away from the forcing must originate from the transport of energy through the surface delimiting the control volume. Since a non-trivial stationary state cannot be described by the homogeneous non-forced 
KHM equation (6), which contains no source term, we must consider explicitly the effects of the inhomogeneities in the following.

We consider here the inhomogeneous generalization of the KHM equation proposed by Hill. ${ }^{44}$ We briefly recall the derivation of this equation, with the addition of the Coriolis force. Let us start from the incompressible Navier-Stokes (NS) equation in the rotating frame

$$
\partial_{t} \mathbf{u}+(\mathbf{u} \cdot \nabla) \mathbf{u}=-\boldsymbol{\nabla} p-2 \mathbf{\Omega} \times \mathbf{u}+\nu \boldsymbol{\nabla}^{2} \mathbf{u},
$$

with $p$ the pressure modified by the centrifugal force and normalized by the fluid density. Taking the difference between points $\mathbf{x}_{B}=\mathbf{X}+\mathbf{r} / 2$ and $\mathbf{x}_{A}=\mathbf{X}-\mathbf{r} / 2$ and taking the scalar product with $\delta \mathbf{u}=\mathbf{u}_{B}-\mathbf{u}_{A}$ yield

$$
\begin{aligned}
\partial_{t}(\delta \mathbf{u})^{2}+\nabla_{\mathbf{r}} \cdot(\delta \mathbf{u})^{2} \delta \mathbf{u}= & 2 v \nabla_{\mathbf{r}}^{2}(\delta \mathbf{u})^{2}-4 \tilde{\epsilon} \\
& +\nabla_{\mathbf{X}} \cdot\left[-(\delta \mathbf{u})^{2} \tilde{\mathbf{u}}-2 \delta p \delta \mathbf{u}+\frac{v}{2} \nabla_{\mathbf{X}}\left((\delta \mathbf{u})^{2}-8 \tilde{\mathbf{\tau}}\right)\right],
\end{aligned}
$$

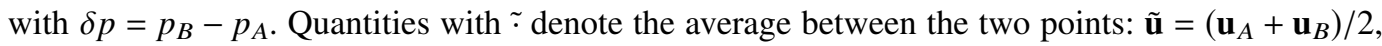
$\tilde{p}=\left(p_{A}+p_{B}\right) / 2$, and $\tilde{\epsilon}=\left(\epsilon_{A}+\epsilon_{B}\right) / 2$, with $\epsilon=\frac{v}{2}\left(\partial_{j} u_{i}+\partial_{i} u_{j}\right)^{2}$ the local energy dissipation rate. The last term of the equation involves the velocity correlation tensor $\tilde{\boldsymbol{\tau}}=\left(\boldsymbol{\tau}_{A}+\boldsymbol{\tau}_{B}\right) / 2$, with $\tau_{i j}$ $=u_{i} u_{j}$. Importantly, all the terms in Eq. (12) are functions of $(\mathbf{X}, \mathbf{r}, t)$, and the nonlinear term splits into a scale-to-scale transfer term (divergence with respect to separation $\mathbf{r}$ ) and a transport term (divergence with respect to mid-point $\mathbf{X}$ ).

We consider both the spatial average $\langle\cdot\rangle_{\mathbf{X}}$ over a control volume $V_{X}$ and the ensemble average $\langle\cdot\rangle_{E}$ of Eq. (12). Using the divergence theorem to express the inhomogeneous terms as a flux through the closed surface $S_{X}$ delimiting the control volume $V_{X}$, we obtain

$$
\partial_{t}\left\langle(\delta \mathbf{u})^{2}\right\rangle_{\mathbf{X}, E}+\nabla_{\mathbf{r}} \cdot\left\langle(\delta \mathbf{u})^{2} \delta \mathbf{u}\right\rangle_{\mathbf{X}, E}=2 v \nabla_{\mathbf{r}}^{2}\left\langle(\delta \mathbf{u})^{2}\right\rangle_{\mathbf{X}, E}-4\langle\tilde{\epsilon}\rangle_{\mathbf{X}, E}+\Phi_{\text {inh }}(\mathbf{r}),
$$

where the flux term writes

$$
\Phi_{\mathrm{inh}}(\mathbf{r})=\frac{1}{V_{X}} \oint_{S_{X}}\left(-\left\langle(\delta \mathbf{u})^{2} \tilde{\mathbf{u}}\right\rangle_{E}-2\langle\delta p \delta \mathbf{u}\rangle_{E}+\frac{v}{2} \nabla_{\mathbf{X}}\left\langle(\delta \mathbf{u})^{2}-8 \tilde{\mathbf{\tau}}\right\rangle_{E}\right) \cdot d \mathbf{S}_{\mathbf{X}}
$$

The unit vector, $d \mathbf{S}_{\mathbf{X}}$, is directed outward of the control volume by convention. In the scale-by-scale budget (13), the energy input (or output) at a given scale $\mathbf{r}$ is ensured by the term $\Phi_{\text {inh }}(\mathbf{r})$, which originates from the inhomogeneities in the pressure and velocity statistics. For homogeneous turbulence, one has $\langle\tilde{\epsilon}\rangle_{\mathbf{X}, E}=\langle\epsilon\rangle_{\mathbf{x}, E}$ and $\Phi_{\text {inh }}=0$, so Eq. (13) becomes the usual KHM equation (6).

The flux term (14) contains three contributions:

(i) $\oint-\left\langle(\delta \mathbf{u})^{2} \tilde{\mathbf{u}}\right\rangle_{E} \cdot d \mathbf{S}_{\mathbf{X}}$ is the flux of cumulative energy $(\delta \mathbf{u})^{2}$ through the surface $S_{X}$ due to advection by the velocity $\tilde{\mathbf{u}}=\left(\mathbf{u}_{A}+\mathbf{u}_{B}\right) / 2$. It is positive when $\tilde{\mathbf{u}}$ is directed into the control volume. Note that this term takes a simple form in the classical configuration of a wind-tunnel: the transport velocity $\tilde{\mathbf{u}}$ is essentially replaced by the uniform mean velocity $\mathbf{U}_{\mathbf{0}}$, and the energy flux per unit surface becomes $\oint-\left\langle(\delta \mathbf{u})^{2}\right\rangle_{E} \mathbf{U}_{\mathbf{0}} \cdot d \mathbf{S}_{\mathbf{X}}$. The inward flux of $(\delta \mathbf{u})^{2}$ through the upstream face of the control volume is larger than the outward flux through the downstream face, hence a net flux of kinetic energy into the control volume, which is dissipated at the same rate by viscosity. By contrast, the time-averaged velocity is negligible in the present experiment when $\Omega>0$, and energy input in the control volume proceeds through advection of turbulent kinetic energy by the turbulent velocity itself.

(ii) The term $\oint-2\langle\delta p \delta \mathbf{u}\rangle_{E} \cdot d \mathbf{S}_{\mathbf{X}}$ originates from the work of the pressure force through the boundary of the control volume. This term cannot be measured experimentally. However, under the assumption of local axisymmetry and homogeneity at small scale, this term is expected to be much smaller than the advection term for scales much smaller than the characteristic scale of inhomogeneity (see Appendix).

(iii) The diffusion term in Eq. (14) is weak because it corresponds to derivatives with respect to $\mathbf{X}$, which are negligible compared to derivatives with respect to $\mathbf{r}$ at small scales for weakly inhomogeneous turbulence (see Appendix). 
We stress the fact that the present inhomogeneous KHM equation has a clear interpretation for scales smaller than the characteristic scale of inhomogeneity, for which the contribution from the pressure can be neglected. This is because the two-point velocity-pressure correlation can be written either as a divergence with respect to $\mathbf{X}$ or with respect to $\mathbf{r}$, so the interpretation of $\Pi$ as the only scale-by-scale transfer term becomes questionable when the velocity-pressure correlation is significant, i.e., for large (inhomogeneous) scales. In the following, we show that interesting modifications of the energy transfers by global rotation occur in the range of scales for which the velocity-pressure correlation is indeed negligible.

\section{B. Scale-by-scale budget for 2D3C flows}

For rapid global rotation, the flow becomes weakly dependent on the vertical coordinate far from the horizontal boundaries. In the following, we assume a purely $2 \mathrm{D} 3 \mathrm{C}$ velocity field in the bulk of the flow, and write separate equations for the evolution of the horizontal and vertical energies. In this 2D limit, the same analysis as in Sec. V A, but performed here on the horizontal components of Eq. (11) only, yields

$$
\partial_{t}\left\langle\left(\delta \mathbf{u}_{\perp}\right)^{2}\right\rangle_{\mathbf{X}, E}+\nabla_{\mathbf{r}} \cdot\left\langle\left(\delta \mathbf{u}_{\perp}\right)^{2} \delta \mathbf{u}_{\perp}\right\rangle_{\mathbf{X}, E}=2 v \nabla_{\mathbf{r}}^{2}\left\langle\left(\delta \mathbf{u}_{\perp}\right)^{2}\right\rangle_{\mathbf{X}, E}-4\left\langle\tilde{\epsilon}_{\perp}^{(\perp)}\right\rangle_{\mathbf{X}, E}+\Phi_{\mathrm{inh}}^{(\perp)}(\mathbf{r})
$$

with

$$
\Phi_{\mathrm{inh}}^{(\perp)}(\mathbf{r})=\frac{1}{V_{X}} \oint_{S_{X}}\left(-\left\langle\left(\delta \mathbf{u}_{\perp}\right)^{2} \tilde{\mathbf{u}}_{\perp}\right\rangle_{E}-2\left\langle\delta p \delta \mathbf{u}_{\perp}\right\rangle_{E}+\frac{v}{2} \nabla_{\mathbf{X}}\left\langle\left(\delta \mathbf{u}_{\perp}\right)^{2}-8 \tilde{\mathbf{\tau}}_{\perp}\right\rangle_{E}\right) \cdot d \mathbf{S}_{\mathbf{X}}
$$

where the subscript $\perp$ in $\delta \mathbf{u}_{\perp}, \tilde{\mathbf{u}}_{\perp}$, and $\tilde{\boldsymbol{\tau}}_{\perp}$ indicates that only the horizontal velocity components are considered, and the $\sim$ indicates that the quantity is a mid-point average. This equation is an inhomogeneous version of the KHM equation for the horizontal flow only. Similarly, from the vertical component of the NS equation, one can also compute the budget for the vertical energy,

$$
\partial_{t}\left\langle\left(\delta u_{\|}\right)^{2}\right\rangle_{\mathbf{X}, E}+\nabla_{\mathbf{r}} \cdot\left\langle\left(\delta u_{\|}\right)^{2} \delta \mathbf{u}_{\perp}\right\rangle_{\mathbf{X}, E}=2 v \nabla_{\mathbf{r}}^{2}\left\langle\left(\delta u_{\|}\right)^{2}\right\rangle_{\mathbf{X}, E}-4\left\langle\tilde{\epsilon}_{\perp}^{(\|)}\right\rangle_{\mathbf{X}, E}+\Phi_{\mathrm{inh}}^{(\|)}(\mathbf{r})
$$

with

$$
\Phi_{\mathrm{inh}}^{(\|)}(\mathbf{r})=\frac{1}{V_{X}} \oint_{S_{X}}\left(-\left\langle\left(\delta u_{\|}\right)^{2} \tilde{\mathbf{u}}_{\perp}\right\rangle_{E}+\frac{v}{2} \nabla_{\mathbf{X}}\left\langle\left(\delta u_{\|}\right)^{2}-8 \tilde{\tau}_{\|}\right\rangle_{E}\right) \cdot d \mathbf{S}_{\mathbf{X}}
$$

where the subscript $\|$ refers to the vertical component of the velocity. This equation is an inhomogeneous generalization of Yaglom's equation for a passive scalar field: ${ }^{42,54}$ the tracer $u_{\|}$enters and leaves the control volume through advection by the horizontal velocity $\tilde{\mathbf{u}}_{\perp}$. Inside the control volume, nonlinearities transfer the vertical energy between different scales $\mathbf{r}$ through stretching and folding by the horizontal field, and viscosity damps the strong gradients created by these processes. We stress the fact that Eq. (17) does not involve pressure: all the terms in this equation can be accessed through stereo-PIV measurements in a horizontal plane.

\section{Experimental assessment of the horizontal and vertical kinetic energy budget}

In the following, we focus on the highest rotation rate, $\Omega=16 \mathrm{rpm}(R o=0.068)$, for which we expect the turbulent flow to reach a quasi-2D3C state, so that we can apply the $\perp$ vs. \| decomposition of the inhomogeneous KHM equation derived above. Note that in this case, the ensemble-averaged flow is negligible (see Table I), so we simply consider $\mathbf{u}=\mathbf{u}^{\prime}$.

We consider for the control domain a centered square in the square PIV field, with a maximum separation $r_{\perp}=60 \mathrm{~mm}$. One can think about the corresponding control volume as a parallelepiped of arbitrary vertical length, with zero fluxes through the top and bottom boundaries. Because turbulence is stationary in the experiment, we replace the ensemble average $\langle\cdot\rangle_{E}$ by a temporal one, which we denote $\div$. 


\section{Horizontal kinetic energy budget}

Let us first consider the energy budget for the horizontal kinetic energy. In statistically steady state, Eq. (15) writes

$$
\tilde{\epsilon}_{\perp}^{(\perp)}=-\Pi_{\perp}^{(\perp)}+D_{\perp}^{(\perp)}+A_{\perp}^{(\perp)}+W_{p}+N T^{(\perp)}
$$

with

$$
\begin{array}{cc}
\epsilon_{\perp}^{(\perp)}=\frac{v}{2}\left\langle\overline{\left(\partial_{\alpha} u_{\beta}+\partial_{\beta} u_{\alpha}\right)^{2}}\right\rangle, & \left.D_{\perp}^{(\perp)}=\frac{v}{2} \nabla_{\perp}^{2} \overline{\left\langle\left(\delta \mathbf{u}_{\perp}\right)^{2}\right.}\right\rangle \\
A_{\perp}^{(\perp)}=-\frac{1}{4 V_{X}} \oint_{S_{X}} \overline{\left(\delta \mathbf{u}_{\perp}\right)^{2} \tilde{\mathbf{u}}_{\perp}} \cdot d \mathbf{S}_{\mathbf{X}}, & W_{p}=-\frac{1}{2 V_{X}} \oint_{S_{X}} \overline{\delta p \delta \mathbf{u}_{\perp}} \cdot d \mathbf{S}_{X} .
\end{array}
$$

For sake of simplicity, $\left\langle\overline{\tilde{\epsilon}_{\perp}^{(\perp)}}\right\rangle$ and $\left\langle\overline{\epsilon_{\perp}^{(\perp)}}\right\rangle$ will be simply noted as $\tilde{\epsilon}_{\perp}^{(\perp)}$ and $\epsilon_{\perp}^{(\perp)}$ in the following.

The horizontal flux of horizontal energy $\Pi_{\perp}^{(\perp)}$ is defined in Eq. (9), and $\epsilon_{\perp}^{(\perp)}$ is the dissipation of the horizontal velocity by the horizontal shearing (with summation over $\alpha, \beta=x, y$ ), such that $D_{\perp}^{(\perp)}\left(r_{\perp} \rightarrow 0\right)=\epsilon_{\perp}^{(\perp)}$. In Eq. (19), $N T^{(\perp)}$ contains the viscous contribution from inhomogeneities, which we neglect in the following (it is at least 2 orders of magnitude smaller than the other terms), and the vertical transport, which we cannot compute from 2D3C measurements. Here, $\langle$. still denotes the spatial average over $\mathbf{X} \in V_{X}$. Under the assumption of weak inhomogeneity, the velocity-pressure flux, $W_{p}$, is expected to be small compared to the transport $A_{\perp}^{(\perp)}$ (see Appendix), and the remaining terms in Eq. (19) can be readily computed from the 2D-3C PIV measurements.

Figure 7(a) shows the three measurable terms of the rhs of Eq. (19) together with their sum, $S^{(\perp)}=-\Pi_{\perp}^{(\perp)}+D_{\perp}^{(\perp)}+A_{\perp}^{(\perp)}$. These terms are averaged over the azimuthal angle $\varphi$. We observe a good agreement between $\tilde{\epsilon}_{\perp}^{(\perp)}$ and the sum $S^{(\perp)}$ for scales smaller than $40 \mathrm{~mm}$, to within $20 \%$. The approximation of locally homogeneous and axisymmetric turbulence, with a negligible velocitypressure flux $W_{p}$, thus seems valid at small scales. For larger scales, we observe a significant departure between $\tilde{\epsilon}_{\perp}^{(\perp)}$ and $S^{(\perp)}$, up to a factor of 2 at $r_{\perp} \simeq 60 \mathrm{~mm}$ : for such large scales, the turbulent flow cannot be considered as locally homogeneous anymore; the non-measured pressure term $W_{p}$ cannot be neglected, so the interpretation of $\Pi_{\perp}^{(\perp)}$ as a scale-by-scale energy transfer becomes incorrect.

Focusing on scales smaller than $40 \mathrm{~mm}$, the advection of horizontal kinetic energy $A_{\perp}^{(\perp)}$ is the only source term in the energy budget. This source term is maximum at large scales, as expected, but it remains significant over the whole range of scales, suggesting a broad-band energy injection in this system. If $E\left(r_{\perp}\right)$ is interpreted as a cumulative energy for eddies of horizontal scale $r_{\perp}$ or less, the scale-by-scale energy density has the form $d E / d r_{\perp}$, so the corresponding forcing density is $d A_{\perp} / d r_{\perp}$. Since we observe $A_{\perp} \simeq r_{\perp}^{1.5}$ at intermediate scales, the forcing density scales as $r_{\perp}^{0.5}$, and it remains significant over the range of scales considered here. This broad-band energy injection is an important feature of boundary-forced inhomogeneous turbulence and is in strong contrast with the narrow-band forcing often considered in numerical simulations of homogeneous rotating turbulence.

Although the inverse cascade is evident from this horizontal energy budget, it must be noted that its magnitude remains moderate: $\Pi_{\perp}^{(\perp)}$ is never the dominant contribution to the budget, even at the crossover between the viscous diffusion and the forcing. A well developed inverse cascade over a wide range of quasi-homogeneous scales at much larger Reynolds number would be characterized by $\Pi_{\perp}^{(\perp)} \simeq A_{\perp} \gg \tilde{\epsilon}_{\perp}^{(\perp)}$. Here, the amount of energy transferred to large scales remains at the best of the same order as the small-scale viscous dissipation $\tilde{\epsilon}_{\perp}^{(\perp)}$.

\section{Vertical kinetic energy budget}

We now consider the vertical kinetic energy budget using Eq. (17) in statistically steady state

$$
\tilde{\epsilon}_{\perp}^{(\|)}=-\Pi_{\perp}^{(\|)}+D_{\perp}^{(\|)}+A_{\perp}^{(\|)}+N T^{(\|)}
$$



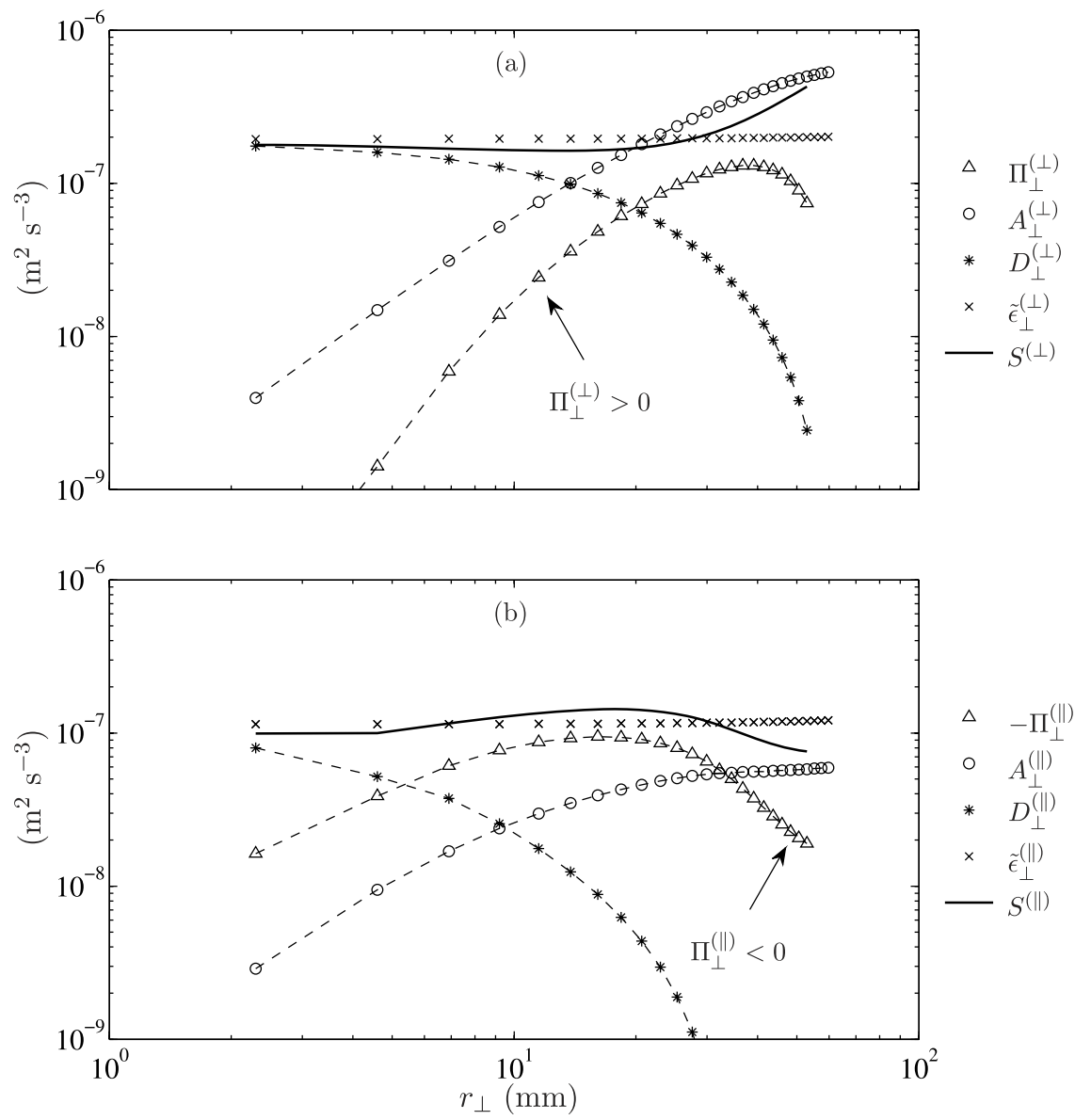

FIG. 7. Scale-by-scale energy budget in the horizontal plane, for (a) the horizontal kinetic energy $\left(\delta \mathbf{u}_{\perp}^{\prime}\right)^{2}$ and (b) the vertical kinetic energy $\left(\delta u_{\|}^{\prime}\right)^{2}$ : Horizontal flux $\Pi_{\perp}^{(\perp)}$ (respectively, $\left.\Pi_{\perp}^{(\|)}\right)$, transport $A_{\perp}^{(\perp)}$ (respectively, $\left.A_{\perp}^{(\|)}\right)$, diffusion $D_{\perp}^{(\perp)}$ (respectively, $D_{\perp}^{(\|)}$), and dissipation rate $\tilde{\epsilon}_{\perp}^{(\perp)}$ (respectively, $\tilde{\epsilon}_{\perp}^{(\|)}$) of horizontal energy (respectively, vertical energy). $S^{(\perp)}$ $=-\Pi_{\perp}^{(\perp)}+D_{\perp}^{(\perp)}+A_{\perp}^{(\perp)}$ and $S^{(\|)}=-\Pi_{\perp}^{(\|)}+D_{\perp}^{(\|)}+A_{\perp}^{(\|)}$are the sums of the measurable right-hand side terms in the energy budgets (19) and (22), respectively. In (a), the horizontal flux of horizontal energy is inverse, from small to large scales $\left(\Pi_{\perp}^{(\perp)}>0\right)$, whereas in (b) the horizontal flux of vertical energy is direct, from large to small scales $\left(\Pi_{\perp}^{(\|)}<0\right)$.

where

$$
\begin{gathered}
\epsilon_{\perp}^{(\|)}=v\left\langle\overline{\left(\nabla_{\perp} u_{\|}\right)^{2}}\right\rangle, \quad D_{\perp}^{(\|)}=\frac{v}{2} \nabla_{\perp}^{2}\left\langle\overline{\left(\delta u_{\|}\right)^{2}}\right\rangle, \\
A_{\perp}^{(\|)}=-\frac{1}{4 V_{X}} \oint_{S_{X}} \overline{\left(\delta u_{\|}\right)^{2} \tilde{\mathbf{u}}_{\perp}} \cdot d \mathbf{S}_{\mathbf{X}} .
\end{gathered}
$$

For sake of simplicity, $\left\langle\overline{\tilde{\epsilon}_{\perp}^{(I)}}\right\rangle$ and $\left\langle\overline{\epsilon_{\perp}^{(\|)}}\right\rangle$will be simply noted as $\tilde{\epsilon}_{\perp}^{(\|)}$and $\epsilon_{\perp}^{(\|)}$in the following.

The horizontal flux of vertical energy $\Pi_{\perp}^{(\|)}$is defined in Eq. (9) and $\epsilon_{\perp}^{(\|)}$is the dissipation of the vertical velocity by the horizontal shearing, with $D_{\perp}^{(\|)}\left(r_{\perp} \rightarrow 0\right)=\epsilon_{\perp}^{(\|)}$. The viscous contribution from inhomogeneities $N T^{(\|)}$is once again discarded for simplicity. A key feature of Eq. (22) is that it does not involve pressure: All the terms can therefore be readily measured from stereo-PIV measurements and the equation should be satisfied for scales at which quasi-two-dimensionality holds.

The terms of Eq. (22) are shown in Fig. 7(b), together with the sum of the right-hand side terms $S^{(\|)}=-\Pi_{\perp}^{(\|)}+D_{\perp}^{(\|)}+A_{\perp}^{(\|)}$. Once again, there is a good overall agreement between $\tilde{\epsilon}_{\perp}^{(\|)}$and $S^{(\|)}$ for $r_{\perp} \leq 40 \mathrm{~mm}$, clearly indicating a direct cascade of vertical kinetic energy. The picture here is simpler than the horizontal energy budget: the vertical energy is advected into the control domain 
$\left(A_{\perp}^{(\|)}\right)$, it is transferred by the nonlinearities to smaller scales $\left(\Pi_{\perp}^{(\|)}\right)$, and it is dissipated at small scales by viscosity $\left(D_{\perp}^{(\|)}\right)$. We can assume that the instabilities of the vortex dipoles generated near the flaps and away from the measurement domain are the source of vertical energy at large scale. Note that for very large Reynolds numbers, an inertial range of scales $r_{\perp}$ with constant flux of vertical energy is expected, characterized by $\Pi_{\perp}^{(\|)}\left(r_{\perp}\right)=-\epsilon_{\perp}^{(\|)}$. Although this is not achieved at the moderate Reynolds number of this experiment, we can, nonetheless, define a significant range of scales, centered around $10-20 \mathrm{~mm}$, for which $-\Pi_{\perp}^{(\|)}$is close to $\epsilon_{\perp}^{(\|)}$within $20 \%$.

\section{Large-scale energy dissipation}

For the largest rotation rate, the scenario of a double cascade, towards small scales for the vertical energy and towards large scales for the horizontal energy, is well established from Figs. 7(a) and 7(b), at least for scales $r_{\perp} \leq 40 \mathrm{~mm}$. At larger scales, the departure between $\tilde{\epsilon}_{\perp}^{(\perp)}$ and $S^{(\perp)}$ may originate both from a departure from two-dimensionality or from the non-measured velocity-pressure correlation. Although less pronounced, there is also a discrepancy between $\tilde{\epsilon}_{\perp}^{(\|)}$and $S^{(\|)}$at large scale, which indicates a departure from a pure 2D3C state and possibly an influence of the horizontal top and bottom boundaries.

We are therefore left with the following question: what sink of energy absorbs the inverse horizontal energy flux at large scales? A first candidate is Ekman friction on the horizontal boundaries. Assuming that the flow is $2 \mathrm{D} 3 \mathrm{C}$ in the central region of the tank, we consider the laminar scaling for the Ekman layer thickness, $\delta_{E} \simeq \sqrt{v / \Omega}$, and we deduce a typical energy dissipation $\epsilon_{\Omega}=\sqrt{v \Omega} u_{\perp}^{2} / H$, where $H$ is the water depth and $u_{\perp}$ is the characteristic horizontal velocity. For $\Omega=16 \mathrm{rpm}$, we obtain $\epsilon_{\Omega} \simeq 10^{-7} \mathrm{~m}^{2} \mathrm{~s}^{-3}$, which turns out to be of the order of the other terms of Eq. (19) (see Fig. 7). A significant fraction of the input horizontal kinetic energy could therefore be dissipated at large scale through Ekman friction. However, this order of magnitude strongly relies on the boundary layers being laminar, which seems questionable in the present experiment.

As an alternate explanation for the energy sink at large scales, we may invoke a feedback of the large-scale flow on the forcing device; the large-scale flow resulting from the inverse cascade induces large-scale pressure forces that transfer some kinetic energy back to the flaps, reducing the overall energy input in the system. In this scenario, the flaps inject energy at intermediate scales and receive energy from the large-scale flow through the work of the pressure forces. In the framework of Eq. (19), the corresponding sink of energy is taken into account by the spatial flux and pressure terms: energy at large scales is transferred outside of the control volume, towards the flaps. Unfortunately, testing this scenario would require a precise measurement of the energy input by the flaps and is beyond the scope of the present study.

\section{CONCLUSION}

In this paper, we provide experimental evidence of a double energy cascade, direct at small scales and inverse at large scales, in a forced rotating turbulence experiment. Since turbulence is statistically steady, the inverse cascade does not manifest through a temporal growth of kinetic energy, but it is characterized by a change of sign of the scale-by-scale energy flux. As the rotation rate is increased, the inverse cascade becomes more pronounced and spreads down to the smallest scales. As compared to previous experimental observations of an inverse cascade in rotating turbulence, ${ }^{37-39}$ here we provide for the first time a direct scale-by-scale measurement of the energy transfers in the horizontal plane. This allows us to distinguish between the horizontal transfers of vertical and horizontal kinetic energies. At the largest rotation rate, this double cascade of the total energy can be described as the superposition of an inverse cascade of horizontal energy and a direct cascade of vertical energy. This is consistent with the 2D3C dynamics expected in the limit of small Rossby numbers, with the vertical velocity behaving as a passive scalar transported by the horizontal flow.

Contrary to numerical simulations, in which energy is usually supplied by a homogeneous body force acting on a prescribed narrow range of wave numbers, in most experiments and in many 
natural flows, energy is supplied at the boundaries. For a control domain away from those boundaries, energy is advected from the boundaries into the domain. This spatial flux of energy, which is strongly related to the inhomogeneities of the turbulent statistics, results in an effective broad-band energy injection term. In order to interpret the energy transfers in such an experiment, it is therefore necessary to separate the contributions from the spatial transport and from the scale-by-scale transfers. We have performed this analysis using the inhomogeneous generalization of the KHM equation proposed by Hill, ${ }^{44}$ and we have measured directly the energy transport term for scales at which the velocity-pressure correlations can be neglected (quasi-homogeneous approximation). Because of this effective broad-band forcing, the inversion scale, which separates the direct and inverse cascades, is not directly prescribed by the geometry of the forcing device and decreases with the imposed rotation rate. Modelling this inversion scale as a function of the Rossby number and forcing geometry remains an open issue of first interest for flows of oceanic and atmospheric relevance, such as convectively driven rotating flows.

\section{ACKNOWLEDGMENTS}

We acknowledge P. Augier, P. Billant, and J.-M. Chomaz for kindly providing the flap apparatus; and A. Aubertin, L. Auffray, C. Borget, and R. Pidoux for their experimental help. This work is supported by the ANR Grant No. 2011-BS04-006-01 "ONLITUR". The rotating platform "Gyroflow" was funded by the "Triangle de la Physique". FM would like to thank the Institut Universitaire de France for its support.

\section{APPENDIX: LOCALLY HOMOGENEOUS AND AXISYMMETRIC TURBULENCE}

In this appendix, we show how one can neglect the velocity-pressure correlations in the inhomogeneous KHM equations (13)-(15) under the assumptions of local homogeneity and axisymmetry.

Let us first consider that the turbulence is invariant to a reflection with respect to a horizontal plane (non-helical turbulence). Let us then denote as $L_{\mathrm{inh}}$ the typical scale of the inhomogeneity of the turbulence statistics, and focus on small separations $r \ll L_{\text {inh }}$, or equivalently $r / L_{\text {inh }}=\zeta \ll 1$. We decompose the velocity and pressure fields into $\mathbf{u}=\mathbf{V}+\mathbf{v}$ and $p=P+q$, where $\mathbf{V}$ and $P$ contain the large scales of the flow and $\mathbf{v}$ and $q$ are the small-scale fluctuations. One can think about $\mathbf{V}$ and $P$ as coarse-grained versions of the velocity and pressure fields on a scale that is smaller than $L_{\text {inh }}$ but larger than the range of scales, $r$, of interest. Then, $\mathbf{v}=\mathbf{u}-\mathbf{V}$ and $q=p-P$ are the small-scale remainders.

Local axisymmetry and homogeneity consist in assuming that the statistics of $\mathbf{v}$ and $q$ are axisymmetric and homogeneous for separations $r \ll L_{\text {inh. }}$. Let us evaluate the different terms in the integrand of (14) under this assumption.

The pressure term decomposes into

$$
\langle\delta \mathbf{u} \delta p\rangle=\langle\delta \mathbf{V} \delta P\rangle+\langle\delta \mathbf{V} \delta q\rangle+\langle\delta \mathbf{v} \delta P\rangle+\langle\delta \mathbf{v} \delta q\rangle .
$$

Because $P$ and $\mathbf{V}$ evolve on a spatial scale $r \ll L_{\text {inh }}$,

$$
\delta P \simeq \mathbf{r} \cdot \nabla P \sim \frac{r}{L_{\mathrm{inh}}} P=\zeta P .
$$

Similarly, $\delta \mathbf{V} \sim \zeta \mathbf{V}$. Hence, $\langle\delta \mathbf{V} \delta P\rangle=O\left(\zeta^{2}\right),\langle\delta \mathbf{V} \delta q\rangle=O(\zeta)$, and $\langle\delta \mathbf{v} \delta P\rangle=O(\zeta)$. We deal with the term $\langle\delta \mathbf{v} \delta q\rangle$ using the assumption of local homogeneity and axisymmetry: under a rotation of angle $\pi$ around a vertical axis passing through the mid-point $\mathbf{X}$, followed by a reflection with respect to the horizontal plane containing $\mathbf{X}, \mathbf{v}$ becomes $\mathbf{v}^{\prime}, \mathbf{v}^{\prime}$ becomes $-\mathbf{v}, q^{\prime}$ becomes $q$, and $q$ becomes $q^{\prime}$. Hence, $\delta \mathbf{v} \delta q$ becomes $-\delta \mathbf{v} \delta q$, so that on statistical average this quantity vanishes: $\langle\delta \mathbf{v} \delta q\rangle=0$. As a consequence, the velocity-pressure correlation term $(\delta \mathbf{u} \delta p)$ is of order $O(\zeta)$ in the weakly inhomogeneous limit. We therefore expect $(\delta \mathbf{u} \delta p)$ to be negligible compared to the transport term $\left\langle(\delta \mathbf{u})^{2} \tilde{\mathbf{u}}\right\rangle$, which is of order $O\left(\zeta^{0}\right)$. 
The viscous term in the integrand of (14) is of order

$$
v \frac{\left\langle(\delta \mathbf{u})^{2}\right\rangle}{L_{\text {inh }}} \sim \frac{v}{|\tilde{\mathbf{u}}| L_{\text {inh }}}\left\langle(\delta \mathbf{u})^{2} \tilde{\mathbf{u}}\right\rangle .
$$

It is therefore negligible compared to the advective term of the integrand, because the Reynolds number based on $L_{\text {inh }}$ is large. Note that because $r \ll L_{\text {inh }}$, derivatives with respect to $r$ are much larger than derivatives with respect to $X$. In the ensemble average of Eq. (12), the "inhomogeneous" viscous contribution can be estimated as $v \nabla_{\mathbf{X}}^{2}\left((\delta \mathbf{u})^{2}\right) \sim \zeta^{2} v \nabla_{\mathbf{r}}^{2}\left\langle(\delta \mathbf{u})^{2}\right\rangle$, hence it is negligible compared to the "homogeneous" viscous contribution $2 v \nabla_{\mathbf{r}}^{2}\left\langle(\delta \mathbf{u})^{2}\right\rangle$. For weakly inhomogeneous turbulence, one can therefore keep the latter while neglecting the former.

To conclude, the advective term, $\left((\delta \mathbf{u})^{2} \tilde{\mathbf{u}}\right)$, is the dominant term in the integrand of Eq. (14). For weakly inhomogeneous turbulence, one can therefore retain only this term of the integrand, which ensures the injection of kinetic energy into the control volume.

${ }^{1}$ P. Sagaut and C. Cambon, Homogeneous Turbulence Dynamics (Cambridge University Press, Cambridge, 2008).

${ }^{2}$ P. A. Davidson, Turbulence in Rotating, Stratified and Electrically Conducting Fluids (Cambridge University Press, Cambridge, 2013).

${ }^{3}$ F. S. Godeferd and F. Moisy, "Structure and dynamics of rotating turbulence: A review of recent experimental and numerical results," Appl. Mech. Rev. (2015).

${ }^{4}$ U. Frisch, Turbulence - The Legacy of A. N. Kolmogorov (Cambridge University Press, Cambridge, 1995).

${ }^{5}$ R. Kraichnan, "Inertial ranges in two-dimensional turbulence," Phys. Fluids 10, 1417 (1967).

${ }^{6}$ E. Lindborg, "Can the atmospheric kinetic energy spectrum be explained by two-dimensional turbulence?," J. Fluid Mech. 388, 259 (1999).

${ }^{7}$ P. Tabeling, “Two-dimensional turbulence: A physicist approach,” Phys. Rep. 362, 1 (2002).

${ }^{8}$ H. Greenspan, The Theory of Rotating Fluids (Cambridge University Press, 1968).

${ }^{9}$ C. Cambon and L. Jacquin, "Spectral approach to non-isotropic turbulence subjected to rotation,” J. Fluid Mech. 202, 295 (1989).

${ }^{10}$ F. Waleffe, "Inertial transfers in the helical decomposition," Phys. Fluids A 5, 677 (1993).

${ }^{11}$ L. M. Smith and F. Waleffe, "Transfer of energy to two-dimensional large scales in forced, rotating three-dimensional turbulence," Phys. Fluids 11, 1608 (1999).

${ }^{12}$ F. Waleffe, "The nature of triad interactions in homogeneous turbulence," Phys. Fluids A 4, 350 (1992).

${ }^{13}$ G. Bordes, F. Moisy, T. Dauxois, and P.-P. Cortet, "Experimental evidence of a triadic resonance of plane inertial waves in a rotating fluid," Phys. Fluids 24, 014105 (2012).

${ }^{14}$ S. Galtier, "Weak inertial-wave turbulence theory," Phys. Rev. E 68, 015301 (2003).

${ }^{15}$ C. Cambon, R. Rubinstein, and F. S. Godeferd, "Advances in wave turbulence: Rapidly rotating flows," New J. Phys. 6, 73 (2004).

${ }^{16}$ L. Bourouiba, "Model of a truncated fast rotating flow at infinite Reynolds number," Phys. Fluids 20, 075112 (2008).

${ }^{17}$ E. J. Hopfinger, F. K. Browand, and Y. Gagne, "Turbulence and waves in a rotating tank," J. Fluid Mech. 125, 505 (1982).

${ }^{18}$ P. Bartello, O. Métais, and M. Lesieur, “Coherent structures in rotating three-dimensional turbulence,” J. Fluid Mech. 273, 1 (1994).

${ }^{19}$ L. Bourouiba and P. Bartello, "The intermediate Rossby number range and two-dimensional three-dimensional transfers in rotating decaying homogeneous turbulence,” J. Fluid Mech. 587, 139 (2007).

${ }^{20}$ L. Bourouiba, D. N. Straube, and M. L. Waite, "Non-local energy transfers in rotating turbulence at intermediate Rossby number," J. Fluid Mech. 690, 129 (2012).

${ }^{21}$ F. Moisy, C. Morize, M. Rabaud, and J. Sommeria, "Decay laws, anisotropy and cyclone-anticyclone asymmetry in decaying rotating turbulence," J. Fluid Mech. 666, 5 (2011).

${ }^{22}$ P. D. Mininni, A. Alexakis, and A. Pouquet, "Scale interactions and scaling laws in rotating flows at moderate Rossby numbers and large Reynolds numbers," Phys. Fluids 21, 015108 (2009).

${ }^{23}$ P. D. Mininni and A. Pouquet, "Rotating helical turbulence. II. Intermittency, scale invariance, and structures," Phys. Fluids 22, 035106 (2010).

${ }^{24}$ A. Pouquet and P. D. Mininni, "The interplay between helicity and rotation in turbulence: Implications for scaling laws and small-scale dynamics," Philos. Trans. R. Soc., A 368, 1635 (2010), revue sur scaling, mais sans cascade inverse.

${ }^{25}$ A. Pouquet, A. Sen, D. Rosenberg, P. D. Mininni, and J. Baerenzung, "Inverse cascades in turbulence and the case of rotating flows," Phys. Scr. T155, 014032 (2013).

${ }^{26}$ Q. Chen, S. Chen, G. L. Eyink, and D. D. Holm, "Resonant interactions in rotating homogeneous three-dimensional turbulence," J. Fluid Mech. 542, 139 (2005).

${ }^{27}$ L. M. Smith and Y. Lee, "On near resonances and symmetry breaking in forced rotating flows at moderate Rossby number," J. Fluid Mech. 535, 111 (2005).

${ }^{28}$ M. Hossain, "Reduction in the dimensionality of turbulence due to a strong rotation," Phys. Fluids 6, 1077 (1994).

${ }^{29}$ A. Sen, P. D. Mininni, D. Rosenberg, and A. Pouquet, "Anisotropy and nonuniversality in scaling laws of the large-scale energy spectrum in rotating turbulence," Phys. Rev. E 86, 036319 (2012).

${ }^{30}$ L. M. Smith, J. R. Chasnov, and F. Waleffe, “Crossover from two- to three-dimensional turbulence,” Phys. Rev. Lett. 77, 2467 (1996).

${ }^{31}$ E. Deusebio, G. Boffetta, E. Lindborg, and S. Musacchio, "Dimensional transition in rotating turbulence," Phys. Rev. E 90, 023005 (2014). 
${ }^{32}$ P. K. Yeung and Ye Zhou, "Numerical study of rotating turbulence with external forcing," Phys. Fluids 10, 2895 (1998).

${ }^{33}$ B. Gallet and W. R. Young, "A two-dimensional vortex condensate at high Reynolds number," J. Fluid Mech. 715, 359 (2013).

${ }^{34}$ M. Thiele and W.-C. Muller, "Structure and decay of rotating homogeneous turbulence," J. Fluid Mech. 637, 425 (2009).

35 T. Teitelbaum and P. D. Mininni, "The decay of turbulence in rotating flows," Phys. Fluids 23, 065105 (2011).

${ }^{36}$ C. Lamriben, P.-P. Cortet, and F. Moisy, "Direct measurements of anisotropic energy transfers in a rotating turbulence experiment," Phys. Rev. Lett. 107, 024503 (2011).

${ }^{37}$ C. N. Baroud, B. B. Plapp, Z.-S. She, and H. L. Swinney, "Anomalous self-similarity in a turbulent rapidly rotating fluid," Phys. Rev. Lett. 88, 114501 (2002).

${ }^{38}$ C. Morize, F. Moisy, and M. Rabaud, "Decaying grid-generated turbulence in a rotating tank," Phys. Fluids 17, 095105 (2005).

${ }^{39}$ E. Yarom, Y. Vardi, and E. Sharon, "Experimental quantification of inverse energy cascade in deep rotating turbulence," Phys. Fluids 25, 085105 (2013).

40 Y. D. Afanasyev and J. D. C. Craig, "Rotating shallow water turbulence: Experiments with altimetry," Phys. Fluids 25, 106603 (2013).

${ }^{41}$ L. J. A. van Bokhoven, H. J. H. Clercx, G. J. F. van Heijst, and R. R. Trieling, "Experiments on rapidly rotating turbulent flows," Phys. Fluids 21, 096601 (2009).

42 A. S. Monin and A. M. Yaglom, Statistical Fluid Mechanics (MIT Press, Cambridge, 1975), Vol. 2.

${ }^{43}$ E. Lindborg, "Correction to the four-fifths law due to variations of the dissipation," Phys. Fluids 11, 510 (1999).

${ }^{44}$ R. J. Hill, "Exact second-order structure function relationships," J. Fluid Mech. 468, 317 (2002).

${ }^{45}$ R. A. Antonia and P. Burattini, "Approach to the 4/5 law in homogeneous isotropic turbulence," J. Fluid Mech. 550, 175 (2006).

${ }^{46}$ L. Danaila, R. A. Antonia, and P. Burattini, "Comparison between kinetic energy and passive scalar energy transfer in locally homogeneous isotropic turbulence," Phys. D 241, 224 (2012).

${ }^{47}$ L. Danaila, J. F. Krawczynski, F. Thiesset, and B. Renou, "Yaglom-like equation in axisymmetric anisotropic turbulence," Phys. D 241, 216 (2012).

${ }^{48}$ B. Gallet, A. Campagne, P.-P. Cortet, and F. Moisy, "Scale-dependent cyclone-anticyclone asymmetry in a forced rotating turbulence experiment," Phys. Fluids 26, 035108 (2014).

${ }^{49}$ P. Billant and J.-M. Chomaz, "Experimental evidence for a new instability of a vertical columnar vortex pair in a strongly stratified fluid," J. Fluid Mech. 418, 167 (2000).

${ }^{50}$ P. Augier, P. Billant, M. E. Negretti, and J.-M. Chomaz, "Experimental study of stratified turbulence forced with columnar dipoles," Phys. Fluids. 26, 046603 (2014).

${ }^{51}$ DaVis, LaVision GmbH, Anna-Vandenhoeck-Ring 19, Goettingen, Germany.

${ }^{52}$ F. Moisy, PIVMat toolbox for Matlab, http://www.fast.u-psud.fr/pivmat.

${ }^{53}$ P. A. Davidson, Turbulence: An Introduction for Scientists and Engineers (Oxford University Press, Oxford, 2004).

${ }^{54}$ A. M. Yaglom, "On the local structure of a temperature field in a turbulent flow," Dokl. Akad. Nauk SSSR 69(6), 743 (1949)

${ }^{55}$ This anisotropic second order structure function (3) is directly related to the 3D turbulent energy spectrum $e(\boldsymbol{\kappa})$ : $E(\mathbf{r})=4\left(k-\iiint e(\boldsymbol{\kappa}) \mathrm{e}^{i \boldsymbol{\kappa} \cdot \mathbf{r}} d^{3} \boldsymbol{\kappa}\right)$, with $k$ the turbulent kinetic energy and $\boldsymbol{\kappa}$ the wavevector.

${ }^{56}$ The normalization by $E_{0}=\left\langle\overline{\mathbf{u}_{A}^{\prime 2}+\mathbf{u}_{B}^{\prime 2}}\right\rangle_{\mathbf{X}}$ is chosen so that $E(\mathbf{r}) / E_{0}=1$ when an exact decorrelation of $\mathbf{u}_{A}^{\prime}$ and $\mathbf{u}_{B}^{\prime}$ is observed beyond $r_{\perp}=r_{\max }$. Note that normalizing by $2\left\langle\overline{\mathbf{u}^{\prime 2}}\right\rangle_{\mathbf{X}}$ would give very similar results, to within $3 \%$, because of the nearly homogeneous turbulence in the region of interest. 